\title{
Las asociaciones de enfermedades raras: Estructura de sus redes e identificación de los líderes de opinión mediante la técnica del análisis de redes sociales
}

\section{The associations of rare diseases: the structure of their networks and identification of opinion leaders through the technique of social network analysis}

Jesús Pérez Dasilva. Universidad del País Vasco. España.

jesusangel.perez@ehu.eus

$[\mathrm{CV}] \odot \mathrm{G}$

Ma Teresa Santos Diez. Universidad del País Vasco. España.

mariateresa.santos@ehu.eus

[CV] C

Koldobika Meso Ayerdi. Universidad del País Vasco. España.

koldo.meso@ehu.eus

[CV] $6 \mathrm{G}$

Este trabajo forma parte del proyecto "Noticias, redes y usuarios en el sistema híbrido de medios" (RTI2018095775-B-C41)", financiado por el Plan Nacional del I+D+i, del Ministerio de Economía y Competitividad, y por el Fondo Europeo de Desarrollo Regional (FEDER) y del Grupo Consolidado IT1112-16 del Gobierno Vasco.

\section{Cómo citar este artículo / Referencia normalizada}

Pérez Dasilva, J., Santos Diez, M. T. y Meso Ayerdi, K. (2021). Las asociaciones de enfermedades raras: Estructura de sus redes e identificación de los líderes de opinión mediante la técnica del análisis de redes sociales. Revista Latina de Comunicación Social, 79, 175-205 https://www.doi.org/10.4185/RLCS-2021-1498

\section{RESUMEN}

Introducción. Esta investigación ha empleado la técnica del Análisis de Redes Sociales para analizar la estructura de relaciones de red que envuelve en Twitter a las tres federaciones de asociaciones de enfermedades raras más importantes e identificar a los actores clave en sus comunicaciones. Metodología. Se ha utilizado el software NodeXL, con la visualización como un componente clave, para capturar la red de conexiones de las cuentas objeto de estudio, representar sus patrones de interacción y averiguar la posición que ocupan los usuarios dentro de la red. Conclusiones. Los resultados indican que estas asociaciones emplean las redes sociales para sensibilizar, educar e informar sobre las ER y sus problemáticas. Son cuentas muy influyentes con un alto grado de vinculación y una gran capacidad de prescripción debido al interés que despiertan en una parte de la población estas patologías y todo lo que las rodea. 
PALABRAS CLAVES: Análisis de Redes sociales; ARS; Enfermedades Raras; Twitter; NodeXL

\begin{abstract}
Introduction. This research has used the technique of Social Network Analysis to analyze the structure of network relationships that surrounds on Twitter the three more important federations of associations of rare diseases and identify key actors in their communications. Methodology. NodeXL software has been used, with visualization as a key component, to capture the network of connections of the accounts under study, represent their interaction patterns and find out the position occupied by users within the network. Conclusions. The results indicate that these associations use social networks to raise awareness, educate and inform about RD and its problems. They are very influential accounts with a high degree of connection and a great capacity for prescription due to the interest aroused in a part of the population by these pathologies and everything that surrounds them.
\end{abstract}

KEYWORDS: Social Network Analysis; SNA; Rare Diseases; Twitter; NodeXL.

\title{
CONTENIDOS
}

1. Introducción. 2. Estado de la cuestión. 2.1. El análisis de redes sociales y los social media. 2.2. Las enfermedades raras. 2.3. Las federaciones de asociaciones de enfermedades raras. 3. Objetivos. 4. Hipótesis. 5. Metodología. 6. Resultados. 6.1. NORD. 6.2. EURORDIS. 6.3. FEDER. 7. Conclusiones. 8. Referencias bibliográficas

\section{Introducción}

Las redes sociales se han convertido en un actor cada vez más presente en nuestras vidas. Como apuntan O'Keeffe y Clarke-Pearson (2011: 800), una parte importante de nuestra vida social y emocional ya transcurre en Internet -especialmente en las redes sociales- y a través de herramientas como tabletas o teléfonos móviles. Por eso resulta de interés para el mundo académico investigar esas interacciones que se producen online en los social media e identificar a los principales actores que gestionan el flujo de influencia social e información dentro de un sistema relacional. Estos líderes de opinión son calificados como social media influencers (SMIs) (Freberg et al., 2011: 90) y para reconocerlos hay que estudiar la posición y los vínculos con terceros que poseen los integrantes de una red social. El problema es que la mayor parte de las investigaciones que han estudiado los SMIs han mostrado limitaciones al centrarse sólo en cuantificar aspectos como las ocasiones en que un mensaje es compartido o el número de seguidores (Del Fresno, 2014). "Puesto que la influencia es algo esencialmente cualitativo, estas estrategias solo pueden ser aceptadas como meros puntos de partida (Basille, 2009; Straley, 2010). Identificar a los SMIs solo a través de su actividad y/o a través del conteo de seguidores o amigos no es una aproximación válida y conducirá a resultados pobres" (Del Fresno et al., 2016: 29).

Para ello, los investigadores deben lograr hacer visible la red que rodea a una persona u organización, es decir una representación gráfica con todos los lazos relevantes que unen los nodos estudiados. Es ahí donde muestra su utilidad el análisis de redes sociales ya que está relacionado con el desarrollo de herramientas informáticas que permiten recopilar, monitorizar, analizar y visualizar los datos de las redes sociales para descubrir la estructura invisible de las relaciones online, extraer patrones útiles y entender su complejidad (Fan, 2014: 74). Por ejemplo, se ha empleado con éxito en epidemiología para comprender cómo se propagan las enfermedades (Martin et al., 2011; Berkman et al., 2014; Lichoti et al., 2016) o cómo se difunden las informaciones (Pfeffer, 2014; Dhar et al., 2016, Ahmed y Lugovic, 2019). 
Este artículo va a centrarse en Twitter como campo de investigación y va a emplear el análisis de redes sociales (ARS) como instrumento metodológico para identificar a los SMIs y estudiar las estructuras de red que envuelven a tres organizaciones de enfermedades raras: FEDER, EURORDIS y NORD. Se trata de "un método científico orientado a la optimización de la investigación de los medios sociales, sustituyendo a los enfoques anecdóticos (número de amigos, seguidores, gustos, etc.) y aportando resultados significativos en la identificación de los actores claves en la comunicación dentro de las plataformas sociales online" (Del Fresno et al., 2016: 28).

\section{Estado de la cuestión}

\subsection{El análisis de redes sociales y los social media}

Según Liu (2011: 270), el análisis de redes sociales es útil para la web porque Internet "es esencialmente una sociedad virtual, y por lo tanto una red social virtual, donde cada página puede ser considerada como un actor social y cada hipervínculo como una relación. Muchos de los resultados de las redes sociales se pueden adaptar y ampliar para su uso en el contexto web. Las ideas de análisis de redes sociales han sido decisivas en el éxito que han tenido los motores de búsqueda online". En el mismo sentido se manifiestan investigadores como Del Fresno et al. (2016: 29) cuando señalan que el ARS es el instrumento adecuado para identificar de manera científica los modelos de difusión de la información en las plataformas sociales online.

En los últimos diez años, una serie de trabajos académicos han abordado la cuestión de la difusión en las redes online en general, y de los atributos y roles de los social media influyentes (SMI). Siguiendo a Bakshy et al. (2011: 66), los primeros estudios analizaron la difusión de la información entre grupos de blogueros. Poco después Leskovec junto a Adamic investigaron las redes de recomendaciones surgidas ('cascadas de enlaces') en un sitio de comercio online (Leskovec et al., 2007). Luego las pesquisas se extendieron a otras plataformas. Por ejemplo, Sun et al. (2009) van a analizar las redes en las fan page de Facebook y autores como Kwak et al., (2010) se centraron en las redes de Twitter para identificar a los usuarios más influyentes. En el caso que nos ocupa, desde su aparición en 2006 la plataforma de microblogging creada por Jack Dorsey "ha proporcionado un rico conjunto de datos para los investigadores, lo que ha llevado a la publicación de más de mil artículos académicos" (Wiliams et al., 2012: 384). En este sentido, los estudios más recientes sobre Twitter se centran en el análisis de sentimientos (Salinas et al., 2017; Zambrano et al., 2016); la difusión de información en diversos acontecimientos (Lee et al., 2015; Shin et al., 2016; Groshek y Tandoc, 2017), el estudio de los hashtag (Zappavigna, 2015; Fink et al., 2016) o el uso de la plataforma por diferentes colectivos u organizaciones (Einwiller y Steilen, 2015; Meijer y Torenvlied, 2016; Gil de Zúñiga et al., 2016, Hanusch y Bruns, 2017), entre otros aspectos.

\subsection{Las enfermedades raras}

La medicina y la salud es el tema que más interesa a los españoles según la encuesta sobre la Percepción Social de la Ciencia y la Tecnología de 2018 realizada por la Fundación Española para la Ciencia y la Tecnología (FECYT). Además, Internet (40,3\%) y las redes sociales (48\%) son citadas en dicho trabajo como primera fuente de información científica para una gran parte de los ciudadanos, algo que se hace más evidente en lo que se refiere a las enfermedades raras (en adelante ER). En este sentido, ya en 2016, la encuesta del Observatorio Nacional de las Telecomunicaciones y de la Sociedad de la Información (ONSTSI) recogía que 6 de cada 10 españoles utilizaba Internet para informarse sobre salud. 
El concepto de ER fue desarrollado por primera vez en EEUU a mediados de los 80 y se refería a aquellas patologías que tienen una prevalencia baja en la población, son potencialmente mortales y conllevan un grado de discapacidad, de dependencia y un deterioro de la calidad de vida (Posada, Martín, Ramírez y Abaitua 2008:1). Según la Organización Mundial de la Salud (OMS), 300 millones de personas conviven en todo el mundo con alguna de las 7.000 ER catalogadas hasta 2018 (el 7\% de la población mundial). Según la Federación Española de Enfermedades Raras (FEDER), para ser considerada como rara, cada enfermedad específica sólo puede afectar a menos de 5 de cada 10.000 habitantes. En total, estima que en España existen más de 3 millones de personas con enfermedades poco frecuentes (Castillo et al., 2015: 674). En Europa, en cambio, se considera que una enfermedad es rara cuando afecta a 1 persona de cada 2.000. Según la Organización Europea de Enfermedades Raras (EURORDIS) existen 30 millones de personas afectadas. En EEUU se califica como tal si afecta a menos de 200.000 y se estima que hay 30 millones en todo el país.

Son enfermedades difíciles de diagnosticar y que implican un cambio radical en la calidad de vida de los afectados y sus familias (Huerta et al., 2012). Desde el sitio web de FEDER explican que estos enfermos esperan una media de 5 años hasta obtener un diagnóstico (en el 20\% de los casos transcurren 10 o más años) y, mientras en el 40,9\% de los casos, el afectado no recibe ningún apoyo ni tratamiento, el $26,7 \%$ recibe algún tratamiento inadecuado por la falta de conocimientos sobre su patología, y en el $26,8 \%$ de los casos su enfermedad se ha agravado. Además, su situación empeora por la falta de medicamentos adecuados a sus dolencias y el alto coste que supone. Según FEDER, la cobertura de productos sanitarios por la Sanidad Pública es escasa o nula en el 36\% de los casos. Calculan que cada familia destina más de 350 euros mensuales a su enfermedad (adquisición de medicamentos, tratamiento médico, transporte adaptado, asistencia personal y adaptación de la vivienda) y que más del $70 \%$ de los afectados posee el certificado de discapacidad.

En este contexto, "Internet se perfila como medio ideal para que este colectivo pueda llevar a cabo con éxito sus estrategias de comunicación. Es un medio en el que no es necesario tener un elevado presupuesto para realizar una campaña, rompe las barreras geográficas y cualquier contenido llega de forma inmediata al usuario, al que puede acceder un gran porcentaje de la población. Mediante campañas en internet, en redes sociales, campañas de crowfunding o a través de la difusión de vídeos en canales como YouTube, se puede ofrecer información eficaz a afectados y al resto de ciudadanos, además de solicitar apoyo para la investigación" (Castillo, et al., 2015: 675).

Internet y las redes sociales permiten a los afectados de ER superar las barreras geográficas existentes, compartir experiencias e información. "Esta necesidad de compartir información y conocimientos es especialmente relevante en el caso de las enfermedades raras, siendo estas personas las usuarias de Internet más activas debido a las propias características de las enfermedades: su elevado número (alrededor de 7.000), su baja prevalencia, su carácter incapacitante y la grave amenaza a la vida que suponen" (Armayones et al., 2015: 336).

El empleo de Internet y las redes sociales también resulta de interés para los profesionales sanitarios que pueden realizar consultas virtuales, formar, informar y colaborar en estudios e investigaciones (Guo, et al., 2017; Benetoli, Chen, Aslani, 2018).

En lo que se refiere a investigaciones, son numerosos los estudios relacionados con la salud en los medios (Costa, 2008; Dalley, Buunk y Umit, 2009; Roberts y Good, 2010; Schumaker et al., 2014; Jacobs, et al., 2016; Cavaca et al., 2016 y Emerich et al., 2017). Asimismo, otros trabajos se centran en enfermedades específicas como la gripe A (Camacho, 2009); el VIH-sida (Terrón, 2012); la obesidad (Yoo y Kim, 2012); la salud mental (Peñafiel et al., 2014) o la anorexia (Gúzman y Rodríguez, 2016). Por otra parte, también se han llevado a cabo estudios centrados en las ER y los 
medios de comunicación (Díaz, 2007; Solves y Bañon, 2014; Villa et al., 2014 o Sánchez, 2016). Otras investigaciones recientes se centran en las estrategias de comunicación de las organizaciones de pacientes con ER (López-Villafranca, 2016), sus estrategias en Internet (Castillo, López, Carretón, 2015), el uso de redes sociales por las asociaciones de pacientes de ER (Armayones, et al., 2015) y el encuadre de las ER en los medios de comunicación españoles (López y Castillo, 2018).

Por tanto, se trata de un tema de actualidad e interés que contribuye a sensibilizar a la opinión pública "y a dar visibilidad a un grave problema de salud aportando una visión general y diferente del tratamiento otorgado en los diarios de información general sobre las ER" (Santos y Pérez, 2019).

\subsection{Las federaciones de asociaciones de enfermedades raras}

Los medios de comunicación juegan un papel importante en lo que se refiere a la visibilidad de las ER (Adekunle y Adnan, 2016). En este sentido, Castillo, López y Carretón (2015: 676) apuntan: “Internet ha estrechado lazos entre comunidad médica y pacientes, pero es necesario lograr un nuevo reto, que es el que se plantean las organizaciones de pacientes con el resto de los usuarios". Estas entidades como FEDER, EURORDIS o NORD, ante la realidad de que la población desconoce la existencia de la mayoría de estas patologías, tratan de educar e informar sobre ellas, sensibilizar sobre sus problemáticas "haciendo visibles sus necesidades comunes y proponiendo soluciones para mejorar su calidad de vida, representando sus intereses, defendiendo sus derechos y promoviendo mejoras concretas para lograr su plena inclusión social", tal y como detalla FEDER a través de su web (http://www.enfermedades-raras.org). Según Armayones et al. (2015: 335) las asociaciones están empleando las redes sociales "para sensibilizar sobre las enfermedades raras en general y sobre la propia en particular, así como para intercambiar contenidos relacionados con el apoyo psicológico, médico y social, la promoción y la difusión de la investigación, y la recaudación de fondos".

Las organizaciones de pacientes, como se ha dicho arriba, junto con las asociaciones, proporcionan consejos y apoyo emocional y terapéutico al compartir experiencias comunes (Huerta et al., 2012) ya que muchas veces los afectados se sienten abandonados por el desconocimiento existente sobre sus patologías entre la comunidad médica. En el ámbito internacional son referencia la estadounidense National Organization for Rare Diseases (NORD) y la European Organization for Rare Diseases (EURORDIS). En España hay que citar a la Federación Española de Enfermedades Raras (FEDER).

- NORD surgió en 1983 cuando se asociaron los líderes de varias organizaciones de pacientes con enfermedades raras en Estados Unidos. Hoy día representa a 280 asociaciones. Está presente en Twitter desde octubre de 2009.

- EURORDIS se creó en 1997 y se define como una alianza no gubernamental de organizaciones de pacientes que representa a 837 organizaciones de afectados por enfermedades raras en 70 países, dando cobertura a más de 4.000 enfermedades. Se unió a Twitter en marzo de 2009.

- FEDER nació en 1999 con el objetivo de ser el altavoz de las más de tres millones de personas que conviven con alguna de estas patologías en España. A lo largo de estos 20 años han pasado de 7 a 337 asociaciones y representan más de 1097 patologías y a más de 95.500 personas. Según su última memoria de actividades publicada, en 2017 ayudaron a 123.719 personas (información y orientación, atención psicológica o asesoría jurídica, entre otras). Está presente en Twitter desde enero de 2009.

La actividad desarrollada a lo largo de estos años por estas organizaciones se ha revelado fundamental a la hora de sensibilizar a la opinión pública, promover la investigación médica y brindar servicios a pacientes y familiares. Algo que manifiestan investigaciones recientes como la de López y Castillo (2018: 136): "Los resultados muestran que ha aumentado la visibilidad y el conocimiento sobre estas 
patologías y se refleja una imagen proactiva de este colectivo gracias a sus iniciativas y reivindicaciones".

\section{Objetivos}

El objetivo genérico de esta investigación es analizar la estructura de relaciones de red tejida alrededor de las cuentas de las federaciones de asociaciones de enfermedades raras española (@FEDER_ONG), europea (@eurordis) y estadounidense (@RareDiseases) mediante la técnica del análisis de redes sociales (ARS). Contiene los siguientes objetivos específicos:

O1- Averiguar la posición que ocupan los usuarios dentro de la red e identificar a los actores principales o SMIs.

O2- Estudiar el tipo de vínculos surgidos en la red.

O3- Investigar las redes semánticas y descubrir patrones ocultos en los tuits.

\section{Hipótesis}

H1- Entre los perfiles que presentan mayor ventaja estructural en la red (relacionado con O1) destacan, por encima de los afectados, las asociaciones, fundaciones, empresas biofarmacéuticas y centros de investigación que emplean las redes para promocionar sus actividades y ofrecer información de utilidad a los afectados.

H2- La actividad llevada a cabo por estas entidades para informar y orientar ha sido fundamental y las ha convertido en un referente con alto grado de compromiso con sus seguidores. Se parte de la hipótesis de que la principal forma de crear interacción que se detecte en la estructura $(\mathrm{O} 2)$ será a través de los vínculos del tipo mención o retuit debido a que los afectados consideran estas publicaciones de gran interés para ser compartidos con terceros

H3- En lo que se refiere a las asociaciones de palabras que crean redes de significados (relacionado con O3), los hilos de conversación más relevantes están relacionados con la difusión de sus campañas o eventos que se organizan para sensibilizar, educar e informar sobre las ER y sus problemáticas o solicitar ayuda para la investigación.

\section{Metodología}

Este artículo forma parte de una línea de investigación sobre la presencia de las enfermedades raras en los medios de comunicación y hace referencia al análisis de la estructura de sus redes mediante la técnica del ARS. Para este artículo se analizaron los perfiles de la Federación Española de Enfermedades Raras (FEDER), la europea Rare Diseases Europe (EURORDIS) y la estadounidense National Organization for Rare Diseases (NORD). En su elección se tuvieron en cuenta criterios como su representatividad -agrupan a la mayoría de asociaciones-, tener una cobertura geográfica diferente o que presentan objetivos comunes en cuanto se refiere a labores de coordinación de pacientes, administración pública, científicos, etc, para mejorar la calidad de la atención a los enfermos.

Para hacer visible la red surgida en torno a estas asociaciones de enfermedades raras objeto de estudio e identificar a los nodos centrales (actores principales o SMIs) se escogió Twitter tomando como referencia investigaciones como la de Del Fresno et al. (2016: 29) donde apuntan que el interés de la plataforma para la investigación reside en que es un "medio en el que se produce un intercambio masivo de comunicación interpersonal y dos tipos de conexiones explícitas clave: retweets (RTs) y menciones y respuestas (MTs). Estos dos tipos de conexiones explícitas son más significativas que las relaciones seguidor/amigo". Para Wu et al. (2011: 706), la red de microblogging ofrece un contexto 
interesante en el que abordar la máxima de Lasswell (quién dice qué a quien), sobre todo porque Twitter permite observar fácilmente los flujos de información entre los usuarios de su aplicación. Wiliams et al. (2012: 385) -cuya investigación localizó casi un millar de textos académicos relacionados con la plataforma- apuntan en la misma dirección y señalan que esa abundante producción científica se debe a que es una plataforma abierta, y esa disponibilidad de mensajes publicados en Twitter proporciona un rico conjunto de datos para investigadores académicos de variadas disciplinas que van desde la estadística hasta la antropología. En la misma línea, Bakshy et al. (2011: 65-66) subrayan que Twitter presenta un perfecto laboratorio natural para el estudio de procesos de difusión porque, a diferencia de otras redes como Facebook, Twitter está expresamente dedicada a difundir información.

Para capturar la red de conexiones de las cuentas@FEDER_ONG, @eurordis y @RareDiseases y visibilizar sus patrones de interacción y/o difusión se empleó el programa NodeXL, "uno de los principales software de análisis de código abierto para análisis y visualización de redes" (Lieberman, 2014: 3), diseñado por el sociólogo Marc Smith para facilitar el aprendizaje de los conceptos y métodos de análisis de redes sociales con la visualización como un componente clave (Hansen et al., 2010: 54; Smith, 2014: 1153) y que ha sido utilizado con éxito en diferentes investigaciones como las de Lichoti et al. (2010) -que proporcionó información sobre la epidemiología de la peste porcina africana-, Verweij (2012) -que examinó las conexiones entre políticos y periodistas-, Choi et al. (2014) -que analizó las discusiones políticas de los usuarios de Twitter de Corea-, Dossis et al. (2015) -que estudió la difusión de varios hashtag durante la reciente crisis griega-, Perez, Meso y Mendiguren (2018) - que investigaron de qué hablan los líderes políticos españoles con los medios de comunicación y periodistas- o Ahmed y Lugovic, (2019) -que estudiaron la difusión de noticias-.

La extracción se realizó a comienzos de febrero de 2019 tomando como referencia la investigación sobre la representación de las ER en la prensa española de Santos y Pérez (2019) que reveló que febrero era el tema con mayor actividad.

El programa representó a cada usuario como un vértice (vertex) o nodo y tradujo como aristas (edges) la relación que los mensajes guardaron entre los usuarios. El software descargó 1.551 vértices y 7.384 relaciones entre vértices que permitieron comprender y visualizar la red tejida alrededor de estas organizaciones.

Tabla 1. Vértices y relaciones

\begin{tabular}{|l|l|l|}
\hline Hablando de: & Relaciones $($ edges) & Vértices (vertex) \\
\hline @ FEDER_ONG & 4.864 & 746 \\
\hline @ eurordis & 1.075 & 323 \\
\hline @ RareDiseases & 1.445 & 482 \\
\hline Total & $\mathbf{7 . 3 8 4}$ & $\mathbf{1 . 5 5 1}$ \\
\hline
\end{tabular}

Fuente: Elaboración propia

Asimismo, esta investigación se fundamentó en el concepto de centralidad, compuesto por un grupo de métricas que miden la importancia de los nodos en una red (Scott, 2012). Tomando como referencia las investigaciones de Hansen et al. (2010) y de Smith (2014), y siguiendo a Lieberman, estas métricas de centralidad se clasificaron en:

Tabla 2. Centralidad 


\begin{tabular}{|ll|}
\hline Centralidad & Interpretación en las redes sociales \\
\hline Intermediación & $\begin{array}{l}\text { ¿Qué probabilidad hay de que esta persona sea la } \\
\text { ruta más directa entre dos personas en la red? }\end{array}$ \\
\hline Cercanía & $\begin{array}{l}\text { ¿Cómo de rápido puede esta persona llegar a todos } \\
\text { en la red? }\end{array}$ \\
\hline Prestigio o vector propio & $\begin{array}{l}\text { ¿Cómo de bien está conectada esta persona con } \\
\text { otras personas bien conectadas? }\end{array}$ \\
\hline
\end{tabular}

Fuente: Lieberman (2014: 8)

- Grado de intermediación (betweenness centrality): Permite medir cómo de importante es un nodo y guarda relación con cómo la posición de un usuario en una red condiciona su acceso a la información. Según Hansen et al. (2010: 150), se calcula como el número de veces en que una persona se encuentra en el camino más corto entre otros dos actores. Si un actor tiene un valor alto, esto significa que una gran cantidad de información no redundante pasa a través de él y lo convierte en un 'puente', un guardián o regulador que puede ejercer un control sobre el flujo de la información. Los actores con un valor alto de intermediación pueden filtrar, distorsionar o bloquear informaciones para otras partes de la red. Por el contrario, los actores con valor bajo en intermediación son los que proporcionan contenidos redundantes.

- Grado de cercanía (closeness centrality): La media de las distancias más cortas desde un nodo hacia todos los demás actores. Puede entenderse como la rapidez con que se propaga una información desde un nodo a todos los demás (Brandes, Borgatti y Freeman, 2016).

- Prestigio o vector propio (eigenvector centrality): Mide la influencia o autoridad de un nodo en una red. Para Hansen et al. (2010: 72) una conexión con un actor popular o principal (bien relacionado) es más importante que una conexión con un solitario. Esta métrica tiene en cuenta no sólo el número de conexiones que presenta un nodo (degree centrality) sino cuántos de esos lazos son con personas que a su vez están bien conectadas.

\section{Resultados}

La cuenta con más seguidores es @FEDER_ONG (29.400), seguida por @ RareDiseases (28.200) y @eurordis (20.700). Esta métrica, que indica el alcance potencial que pueden lograr los mensajes de estas organizaciones, está condicionada por el grado de actividad, el de redifusión de sus mensajes o el de 'me gusta' recibidos. En esta línea, el perfil más activo es el de la federación española con 12,2 tuits diarios, el doble que NORD (5,48 mensajes por día) y seis veces más que EURORDIS (1,98 tuits). Además, los resultados muestran que todas son cuentas con un alto grado de vinculación (engagement) ya que más del $60 \%$ de sus tuits son reenviados a terceros porque sus seguidores los consideran relevantes para ser dados a conocer entre sus amistades. NORD consigue un grado de reenvíos del $70,72 \%$ con una media de retuiteo de 7,61 veces por mensaje. EURORDIS consigue un $62,33 \%$ y 8,09 reenvíos por mensaje. Los mensajes de FEDER también tienen gran éxito entre los usuarios y, aunque ocupa la tercera posición, alcanza una tasa de redifusión de sus contenidos del 61,24\% y 4,4 retuiteos por publicación. Por lo tanto, aproximadamente dos de cada tres de los mensajes de estas organizaciones consiguen una significativa repercusión. Prueba de ello es también el importante número de 'likes' que obtienen las tres cuentas. El 77,94\% de las publicaciones realizadas por la asociación norteamericana son marcados como favoritos por los cibernautas. La federación española le sigue de cerca con un $65,49 \%$ de sus tuits que reciben un 'me gusta'. El 61,43\% de lo que publica EURORDIS también gusta a los usuarios.

\subsection{La Federación de Asociaciones de Enfermedades Raras Norteamericana (NORD)}


La cuenta@RareDiseases fue creada en octubre de 2009 y se describe como "la voz de la comunidad de las enfermedades raras". Cuenta con 28.200 seguidores y ha publicado más de 11.800 mensajes.

\subsubsection{Actores con posiciones más favorables en la red}

La estructura de la red tejida alrededor de NORD obtenida con el software muestra una docena de grupos de acuerdo a sus relaciones. Según el grado de intermediación (betweenness centrality), entre los individuos que proporcionan información no redundante en la red que rodea a la organización norteamericana de ER, llama la atención la presencia de un grupo de cuatro cuentas (@ shashanktyagi05, @LSDSS, @ sudhee26, @thezbfoundation) radicadas en India y Pakistán, entre las que destaca la del neurocientífico Dr. Sudheendra Rao (@sudhee26). Junto a ellas, como 'puentes' del flujo de información, también aparecen los perfiles del Dr. Joan Fallon, director de la compañía biofarmaceútica Curemark (@curemarkceo) o la activista y experta en ER Tracy Zervakis (@ChiariTracy), entre otros. Además, en las conversaciones recogidas durante la investigación también hay que mencionar a otros tres usuarios que funcionaron como intermediarios en las comunicaciones entre los actores que dan forma a la estructura: @ roneawkard (cuyas publicaciones llegan a contener hasta 48 menciones por mensaje), @ionisdisrupts (afectado por una ER) y @ lagunagv (afectado por otra ER).

A la hora de estudiar los patrones de interacción y comunicación en Twitter cuando se habla de NORD, también resulta de interés prestar atención al grado de cercanía, que mide la capacidad de una cuenta para acceder al resto de cuentas de la red. Un perfil con un alto grado de cercanía es importante porque puede conectar rápidamente con el resto (Del Fresno et al., 2014). Son cuentas que, a pesar de tener pocas conexiones, permiten llegar a todos los nodos de la red más rápido que desde cualquier otro punto y, por tanto, constituyen una buena posición para monitorear el flujo de información de toda la estructura (Kuz, et al., 2016).

Las personas que más rápido pueden llegar a otras en la red (ventaja estructural), además de NORD, son las siguientes:

1. @ RareDiseaseDay: Cuenta del Día de las Enfermedades Raras (28 de febrero). Su objetivo es sensibilizar a pacientes, familiares y cuidadores de todo el mundo afectados por ER.

2. @ bsyngap: Fundación Bridge the Gap - SYNGAP Education \& Research Fnd. Para servir, educar y financiar la investigación para las familias que enfrentan los efectos de las mutaciones SYNGAP1.

3. @ 1seiders3: Lydia L. Seiders, embajadora NORD en Maryland. Coordina los 200 miembros de la red en Maryland para crear conciencia sobre los aproximadamente 600,000 pacientes afectados por un trastorno raro.

4. @ncats_nih_gov: Cuenta oficial de Twitter del Centro Nacional para el Avance de las Ciencias Translacionales de los Institutos Nacionales de Salud (NIH).

5. @GlobalGenes: Organización líder de defensa del paciente que trabaja para eliminar los desafíos de las ER que enfrentan 350 millones de personas en todo el mundo.

6. @eurordis: Federación de asociaciones europea. Una alianza de más de 800 organizaciones de pacientes con ER que trabajan juntas para mejorar la vida de los 30 millones de personas que viven con una enfermedad rara en Europa.

7. @ KirkBrazeau: Padre cuyo hijo, Archer, fue diagnosticado de Epidermolisis Bullosa Distrófica Recesiva (RDEB). Su historia se hizo famosa por ser una de las elegidas por NORD para mostrar cómo cambia la vida de una familia y cómo se puede superar con esperanza y amor.

8. @ DrPas84Ele: Doctora Eleonora Passeri, consultora en ciencias y redes sociales. 
Otra de las métricas estudiadas es el grado de autoridad (eigenvector), que guarda relación con la influencia que adquiere un perfil en una red y que, además de contabilizar el número de conexiones de un perfil (grado de centralidad), estudia cuántas son con personas que están bien relacionadas. En este caso, los actores con posiciones más favorables (que tienen una ventaja estructural en la red) son casi los mismos que los obtenidos con el grado de cercanía. Esto sugiere que, en general, no hay grandes desigualdades en la centralidad o poder de la cuenta cuando se mide de esta manera. Cambian las posiciones y aparecen dos nuevos actores a tener en cuenta:

- @ mlweldon5: Monica Dudley Weldon es una conferenciante y activista cuyo hijo Beckett fue uno de los primeros diagnosticados en todo el mundo con SYNGAP1, y desde ese día, no ha dejado de hablar de ello.

- @NIH: Cuenta oficial de Twitter de los Institutos Nacionales de Salud de Estados Unidos.

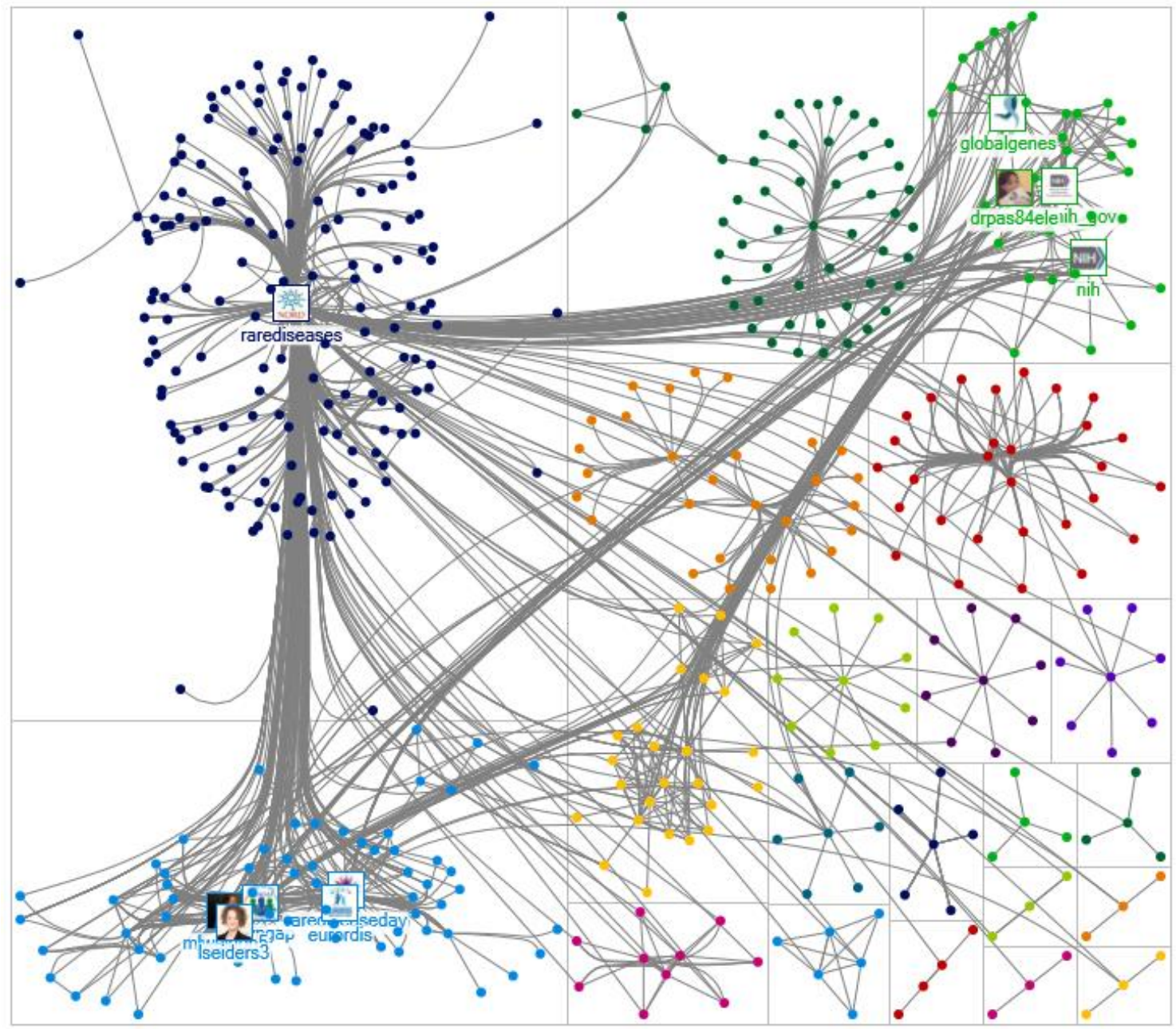

Figura 1: Visualización del grado de autoridad

Fuente: Elaboración propia

\subsubsection{Interacciones en la red}

La red de interacciones que envuelve a la organización norteamericana está compuesta por un 72,66\% de menciones, un $21,45 \%$ de retuits, un 4,295 de respuestas y sólo un 1,59\% por tuits. Predomina la mención como forma mayoritaria de relación con publicaciones como el mensaje de @ runwayofdreams1 a @RareDiseases: 
"I am \#rare. I am one of approximately 100 people in the United States with \#Mucopolysaccharidosis Type VI. Also known as Maroteaux-Lamy (\#MPS-VI). \#RareDisease \#RareDiseaseDay \#showYourStripes \#CareAboutRare@SanfordCoRDS @RareDiseases @GlobalGenes @ rarediseaseday \#MPSVI https://t.co/gtruRq0uux".

Si exceptuamos a NORD los actores más mencionados fueron:

1. @RareDiseaseDay: El 28 de febrero se celebra el día de las enfermedades raras y la investigación se realizó a comienzos de ese mes por lo que no es extraño que esta cuenta acumule una buena cantidad de menciones.

Ejemplo: "Are You Ready!? \#RareDiseaseDay@RareDiseases@ @rarediseasefdn @eurordis @rarediseaseday \#SYNGAP1 \#neverstopping https://t.co/fp06Jn7e9Z" (28/01/2019, 4:36).

2. @eurordis: La Federación de Asociaciones Europea cerró en 2010 un acuerdo de asociación estratégica con NORD estableciendo de esta manera las bases para iniciativas conjuntas clave y una mayor colaboración transatlántica.

Ejemplo: "Did you know that \#RareDiseaseDay was founded by @eurordis in 2008? Learn more abt the international campaign on their Twitter account @ rarediseaseday and visit their website for global campaign resources at https://t.co/scuXryKFZq https://t.co/xqARgwjGLr" (31/01/2019, 21:13:12).

3. @ RareAction: Red americana que lucha a nivel federal por mejorar las vidas de los 30 millones de estadounidenses con ER.

Ejemplo: "Advocates are learning the ins \&amp; outs of the \#orphandrugact Good stuff at \#PArare day today. @RareAction@RareDiseases \#RareDisease \#Pennsylvania https://t.co/svmyj0Kyu2" (01/02/2019, 16:17).

\subsubsection{Temas de conversación más relevantes}

Además de estudiar la estructura de relaciones en red, el software también se ha empleado para analizar las palabras más repetidas en el flujo de información y su asociación con otros términos. De esta manera, se han descubierto los hilos de conversación más relevantes (véase figura 4). La línea de conversación más importante (en azul oscuro) hace referencia al 28 de febrero, día internacional de las ER y se complementa con la segunda asociación más significativa (en azul claro en la imagen).

Ejemplo: "Raise awareness for \#rarediseases! \#ShowYourStripes with NORD on \#RareDiseaseDay, coming up on Thursday, February 28! Learn how to get involved at https://t.co/TfUgm5FA4i https://t.co/XOBI2le0yx" (26/01/2019, 9:55).

El otro hilo de conversación principal se refiere también al día de las enfermedades raras, concretamente a la cebra que es el símbolo de las ER en USA (en color verde oscuro, naranja y rojo).

Ejemplo: "It's \#InternationalZebraDay! DYK that the zebra is the symbol of \#rarediseases in the U.S.? Similar to how each zebra's stripes are unique, each of the 25-30M Americans living w/ \#raredisease are unique. In honor of \#RareDiseaseDay, \#ShowYourStripes! https://t.co/p0g5CEy1W0 https://t.co/eb63sAvnpx" (01/02/2019, 1:21). 


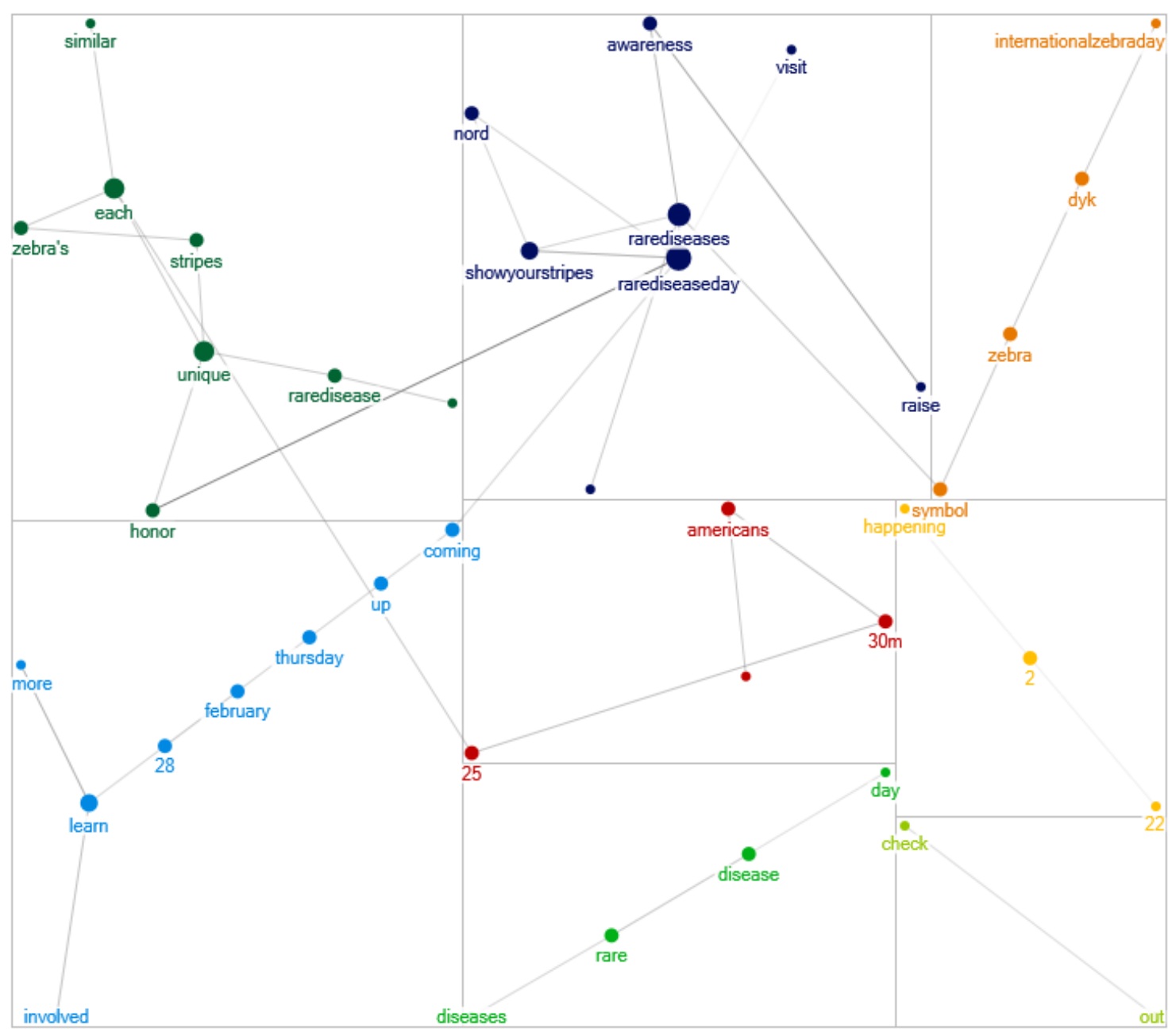

Figura 2: Hilos de conversación más relevantes en la red que rodea a NORD

Fuente: Elaboración propia

\subsection{La Federación de Asociaciones de Enfermedades Raras Europea (EURORDIS)}

La cuenta@eurordis fue creada en marzo de 2009 y se describe como “Una alianza de más de 800 organizaciones de pacientes con enfermedades raras que trabajan juntas para mejorar la vida de los 30 millones de personas que viven con una enfermedad rara en Europa”. Cuenta con 20.700 seguidores y ha publicado más de 4.469 mensajes.

\subsubsection{Actores con posiciones más favorables en la red}

En lo que se refiere al grado de intermediación, en la estructura de relaciones en red que envuelve a EURORDIS los perfiles que tienen mayor ventaja estructural pertenecen a asociaciones y fundaciones deER (@thezbfoundation, @ifsbh, @ERNIthaca y @bsyngap) o a sus dirigentes como Jacqui Browne (@abrihouse) o Marleen aka Sparkles (@ patientexpert). Asimismo, hay que señalar una publicación sobre salud (@ healtheuropa) o la cuenta del día internacional de las ER (@ rarediseaseday). 


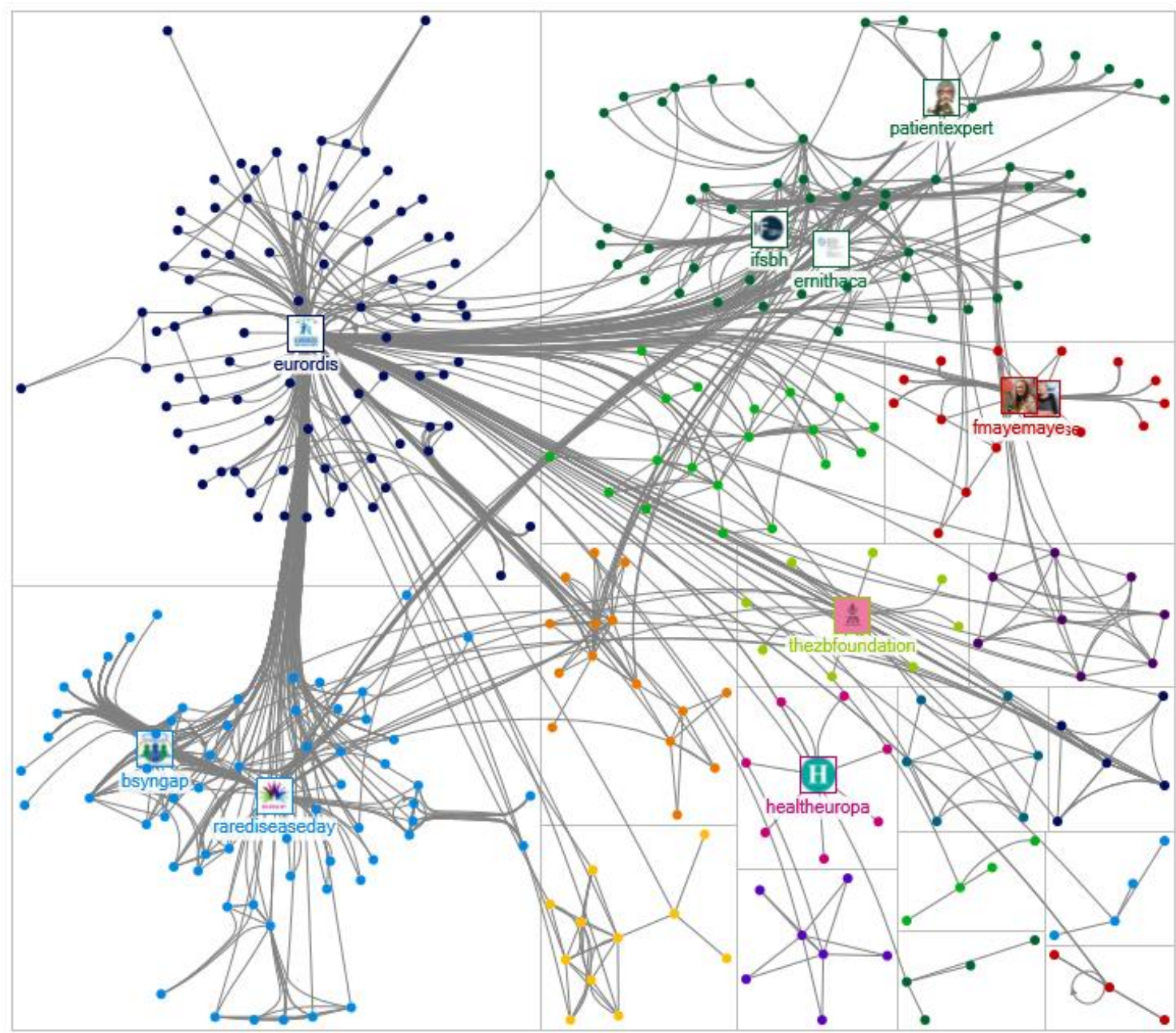

Figura 3: Visualización del grado de intermediación

Fuente: Elaboración propia

Otro aspecto de una posición estructural ventajosa es el grado de cercanía. Las personas que más rápido pueden llegar a otras en la red que envuelve a @eurordis (además de ella) se muestran más abajo. En este sentido, se observa que la mayoría se corresponden con federaciones y redes de referencia de ER. También hay que señalar que la cuenta con mayor grado de cercanía es la cuenta del Día de las ER (@RareDiseaseDay), algo en lo que coincide con la red de NORD y que está relacionado con su celebración el 28 de febrero (cuando se realizó la investigación faltaban tres semanas para su conmemoración). Asimismo, se manifiesta de nuevo la relación entre EURORDIS y la Organización de Enfermedades Raras Norteamericana que, como se ha comentado, unieron fuerzas en el $2010 \mathrm{y}$, entre otras cosas, acordaron promover el Día de las Enfermedades Raras como un evento de reconocimiento internacional.

1. @RareDiseaseDay: Cuenta del Día de las Enfermedades Raras (28 de febrero).

2. @RareDiseases: Federación de asociaciones de ER norteamericana

3. @ERNIthaca: Red de referencia europea sobre malformaciones congénitas y discapacidad intelectual rara (ERN-ITHACA).

4. @ ifsbh: Organización mundial para las asociaciones de Espina Bífida e Hidrocefalia.

5. @ERN_RARE_LIVER: Pacientes con enfermedades hepáticas raras en Europa.

6. @RareDiseasesEU: Ha sido la cuenta de Twitter de 2 Acciones conjuntas de la UE para ER: RD-ACTION y EUCERD JA. 
7. @EU_Health: Comisión de Salud y Seguridad Alimentaria de la UE (SANTE). Trabajan para proteger la salud, prevenir enfermedades y fortalecer los sistemas de salud.

8. @Metab_ERN: La Red Europea de Referencia para las Enfermedades Metabólicas Hereditarias Raras.

9. @ern_reconnet: Red Europea de Referencia sobre Tejido Conectivo Raro y Complejo y Enfermedades Musculoesqueléticas.

En lo que se refiere al grado de prestigio o autoridad, ocho de los actores que ocupaban las primeras posiciones en la métrica de cercanía son también los perfiles con más influencia y que, por tanto, presentan una ventaja estructural en la red tejida alrededor de EURORDIS. Esto indica que no existen grandes desigualdades en la centralidad de la cuenta cuando se mide el grado de prestigio. Cambian algunas posiciones y hay que mencionar a dos nuevos actores:

- @SjogrenpowerAna: Ana Vieira. Paciente con síndrome de Sjögren, que es un trastorno autoinmunitario en el cual se destruyen las glándulas que producen las lágrimas y la saliva. Miembro de La Liga Europea contra el Reumatismo (EULAR).

- @bsyngap: Fundación Bridge the Gap. Relacionada con las mutaciones SYNGAP1. Este es uno de los actores que ocupaba las primeras posiciones en la red de NORD.

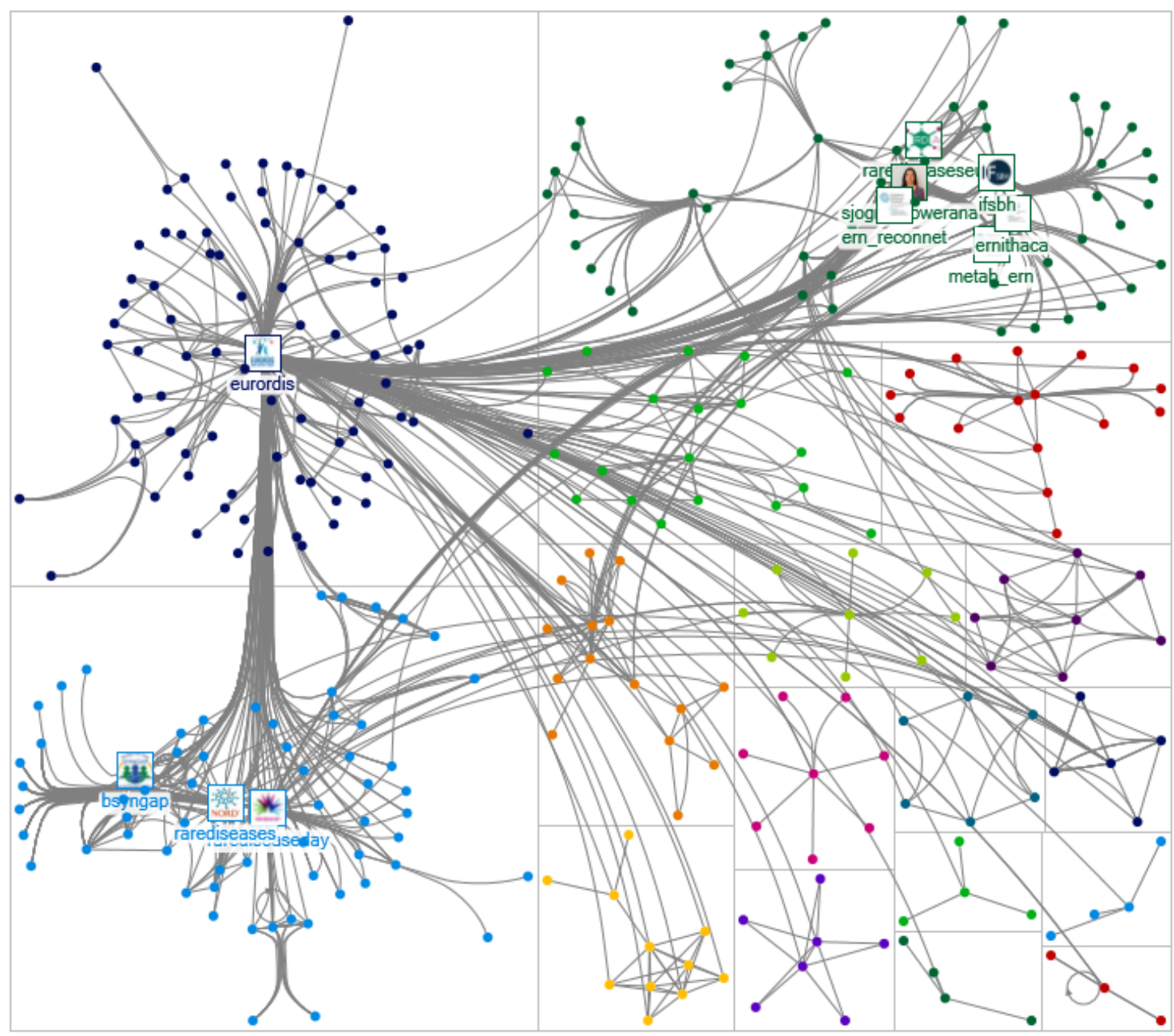

Figura 4: Visualización del grado de autoridad

Fuente: Elaboración propia

\subsubsection{Interacciones en la red surgida en torno a EURORDIS}


Los gráficos anteriores permiten observar fácilmente los flujos de información que se producen entre los usuarios. Un $79,16 \%$ son menciones, un $17,67 \%$ retuits, un $2,79 \%$ respuestas y un $0,37 \%$ tuits. La mención es la forma mayoritaria de relación entre los actores y los más mencionados (sin contar a @ eurordis) fueron:

- @ RareDiseaseDay: Cuenta del Día de las Enfermedades Raras.

Ejemplo: "Did you know that \#RareDiseaseDay was founded by @eurordis in 2008?

Learn more abt the international campaign on their Twitter account @ rarediseaseday and visit their website for global campaign resources at https://t.co/scuXryKFZq https://t.co/xqARgwjGLr" (31/01/2019, 20:50).

- @ RareDiseases: Cuenta de NORD.

Ejemplo: “@GeneticAlliance@GeneticsGSA@_GeneTherapy@TheEllenShow @GlobalGenes@RareDiseases @ eurordis @ rarediseaseday @ RareConnect @ RareDR @ raredisorders @McGill_rareDIG”(31/01/2019,6:42).

- @ rarediseasefdn: Fundación Rare Disease.

Ejemplo: "Are You Ready!? \#RareDiseaseDay@RareDiseases@@rarediseasefdn @eurordis @rarediseaseday \#SYNGAP1 \#neverstopping https://t.co/fp06Jn7e9Z" $(26 / 01 / 2019,17: 45)$.

De nuevo, hay que apuntar las similitudes con la red creada alrededor de NORD. El Día de las ER ocupa también aquí la primera posición, seguido por las menciones a la federación norteamericana (se mencionan entre ellas).

\subsubsection{Temas de conversación más relevantes}

En la red que envuelve a EURORDIS también se han investigado las redes semánticas. Las asociaciones de palabras presentes en los tuits han descubierto diferentes redes de significados. La línea de conversación más importante (en verde claro) se refiere al acceso a terapias para combatir las ER y presenta una conexión muy fuerte con un simposio organizado por la federación europea sobre el tema.

Ejemplo: “Are you a clinician working within a European Reference Network? Register for the 3rd EURORDIS Multi-Stakeholder Symposium to learn more about the crucial role that \#ERNs play in improving access to rare disease therapies. \#RareEU2019 https://t.co/0gxdDQrj8s @ERNIthaca @Metab_ERN https://t.co/UOmIv9KQSe" (26/01/2019, 16:56).

El otro hilo de conversación principal (en verde oscuro y se completa con el azul claro) trata sobre cómo la colaboración entre diferentes redes a nivel de la UE puede marcar una diferencia real en la vida de las personas que viven con una enfermedad rara.

Ejemplo: "The European Reference Networks show that collaborating at EU level can make a real difference to the lives of people living with a rare disease \#ERNs https://t.co/ztxXqyRBuR" $(30 / 01 / 2019,16: 41)$. 


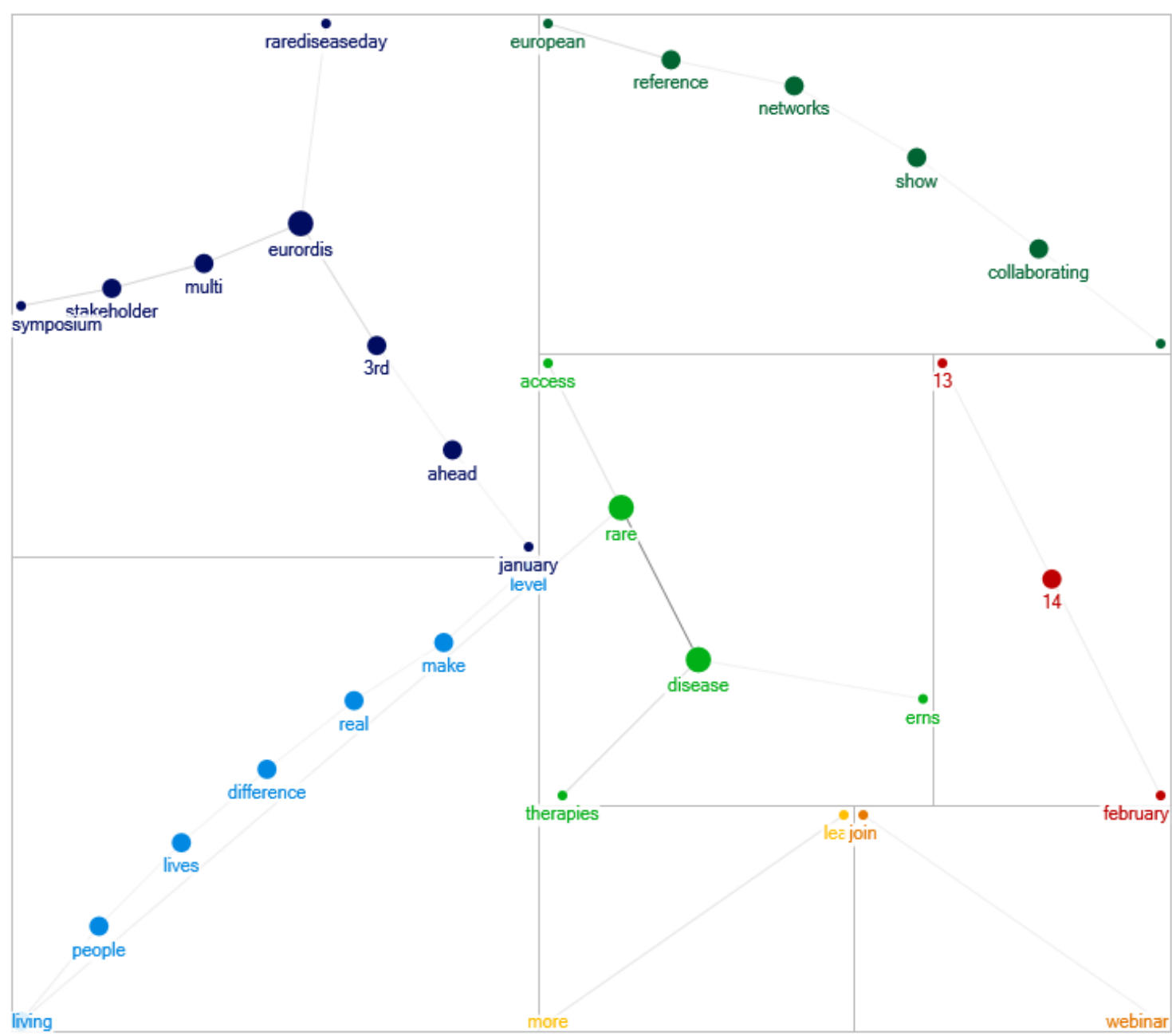

Figura 5: Hilos de conversación más relevantes en la red que rodea a EURORDIS

Fuente: Elaboración propia

\subsection{La Federación Española de Enfermedades Raras}

La cuenta @FEDER_ONG fue creada en enero de 2009, tiene 29.400 seguidores y es el perfil más activo de los tres estudiados, ya que ha publicado más de 63.300 mensajes (seis veces más que NORD y 14 más que EURORDIS).

\subsubsection{Actores con posiciones más favorables en la red}

Como se ha mencionado anteriormente, la red que envuelve a la federación española está compuesta por 746 nodos. Los actores que ocupan las posiciones más favorables en cuanto a su grado de intermediación -es decir, para que un perfil pueda relacionarse con otro con el que no tiene una conexión directa tendría que recurrir a alguno de estos actores intermediarios- son los siguientes: un corredor relacionado con la carrera por la esperanza del 3 de marzo ( @ DavidRumbao); el fundador de la Asociación Nacional de Abogados Matrimonialistas (@CesarMartAbgdo); la fundación Fundación Oír es Clave / AG Bell International (@oiresclave); un pediatra catalán (@ crevertebover); la agencia de comunicación Sombradoble autora de un documental sobre ER (@NoInvisibles); la empresa biofamacéutica Sanofi Genzyme, la tercera más grande del mundo (@RarasReales); una asociación de pacientes (@ffpaciente) o una enfermera (@marisabarcelo), entre otros. Estas cuentas tuvieron un 
valor alto de intermediación por lo que se considera que una gran cantidad de información no redundante pasó a través de ellos.

Si consideramos la red en términos de la cercanía del actor -ya que la cercanía y la distancia supone una ventaja estructural- las cuentas que pueden conectar más rápidamente con el resto en la distribución que envuelve a FEDER (además de ella) son:

1. @NoInvisibles: Cuenta perteneciente al proyecto 'Raras pero no invisibles', que comenzó como un documental para dar visibilidad a las enfermedades minoritarias, las personas y la investigación relacionada. Fue un trabajo realizado desde la agencia Sombradoble que en la actualidad trata de acercar la ciencia, la medicina y la tecnología al público.

2. @RarasReales: Cuenta creada por Sanofi España, empresa que forma parte de Genzyme Corporation, una compañía biotecnológica con sede en Massachusetts y que es considerada la tercera compañía biotecnológica más grande del mundo.

3. @ sanidadgob: Ministerio de Sanidad, Consumo y Bienestar Social.

4. @ffpaciente: Asociación de pacientes.

5. @ nh487: Noah Higón Bellver. Jurista y politóloga afectada por una ER.

6. @Fundacion_ONCE: Fundación de la Organización Nacional de Ciegos Españoles.

7. @ CIBERER: Centro de Investigación Biomédica en Red de \#EnfermedadesRaras (CIBERER), dependiente del Instituto de Salud Carlos III.

8. @ lou_cordon: Lourdes Cordón. Bióloga Ph.D., especializada en citometría de flujo.

9. @espinmesado: David Mesado. Técnico de laboratorio en el Instituto de Investigación Sanitaria La Fe.

En este caso, al contrario que con EURORDIS y NORD, la cercanía del Día Internacional de las ER no alcanza la misma relevancia. En primer lugar aparece el perfil de un documental sobre ER, seguido de una empresa que pertenece a la tercera compañía biotecnológica más grande del mundo. En tercera posición se sitúa el Ministerio de Sanidad. El resto pertenece a asociaciones de pacientes, fundaciones, centros de investigación y sus investigadores o técnicos. 


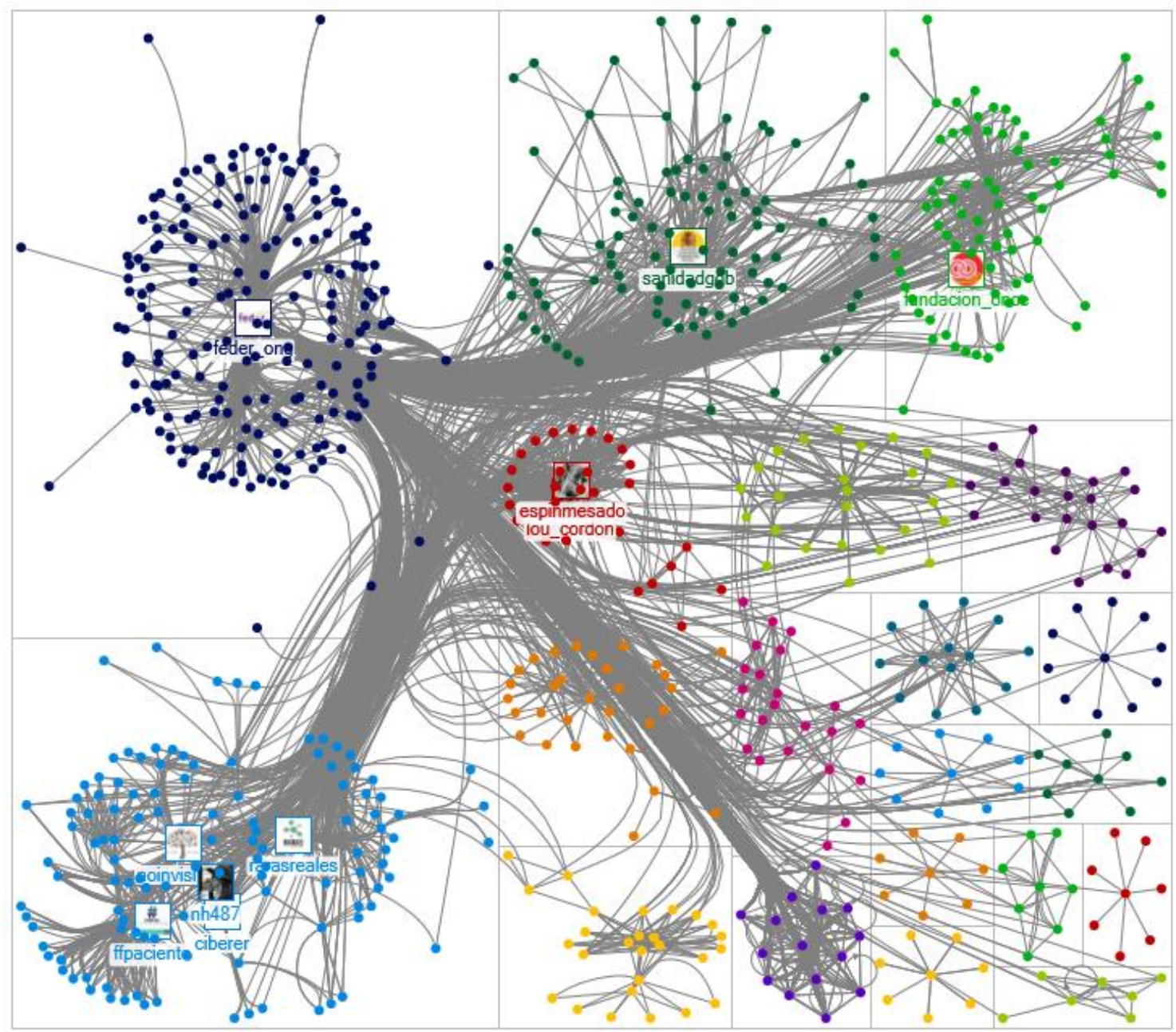

Figura 6: Visualización del grado de cercanía

Fuente: Elaboración propia

Si consideramos la red en términos de prestigio -si un nodo está unido a nodos importantes tendrá un "eigenvector" o "autoridad" alta- las cuentas mejor conectadas en la red que envuelve a FEDER (además de ella) son casi las mismas que tenían mayor grado de cercanía. Esto indica que no existen grandes diferencias en la centralidad de la red cuando se mide el grado de prestigio. Cambian algunas posiciones y aparecen dos nuevos a actores a tener en cuenta:

- @LFdCesmas: La Fuerza del Corazón es una red que reúne las diversas causas sociales que son apoyadas por el cantante Alejandro Sanz, FEDER entre ellas.

- @ilunion: Grupo empresarial de la ONCE para la inserción social de las personas con discapacidad. Cuenta con una plantilla de 32.000 trabajadores. 


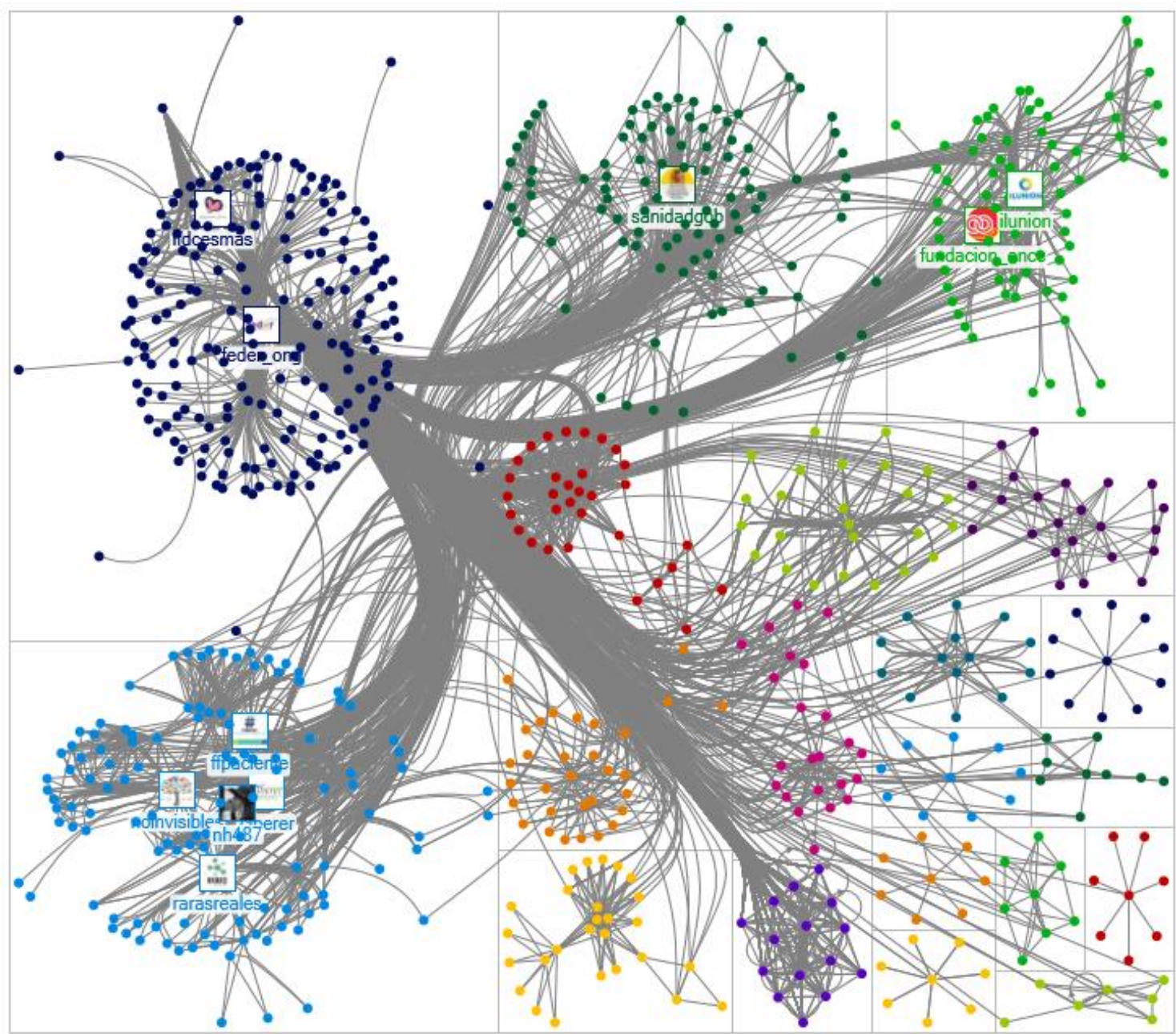

Figura 7: Visualización del grado de autoridad

Fuente: Elaboración propia

\subsubsection{Interacciones en la red surgida en torno a FEDER}

En la cuenta @FEDER_ONG destaca la mención como forma preferida de relación $(73,23 \%)$, seguida por el retuit $(22,03 \%)$, las respuestas $(3,61 \%)$ y los tuits $(1,11 \%)$. Si excluimos la cuenta objeto de estudio, los actores más mencionados fueron:

- @AlejandroSanz: Cantante español que ha vendido millones de discos en todo el mundo y ha ganado varios premios Grammy.

Ejemplo: "Lo estabais esperando y ya está aquí, el cartel oficial de la X Carrera

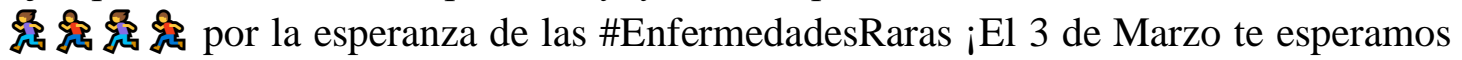
en la Casa de Campo de Madrid! @FEDER_ONG @LFdCesmas @alejandrosanz $\rightarrow$ https://t.co/yDHEl7JWyf https://t.co/YgqtTGFkTx" (27/01/2019, 10:22:23).

- @LfdCesmas: Red que aglutina las diversas causas sociales apoyadas por el cantante Alejandro Sanz, FEDER entre ellas.

Ejemplo: "Están todos invitados @FEDER_ONG \#enfermedadesraras @ AlejandroSanz@VienedelAireARG@LfdCesmas \#JuntosSomosMás https://t.co/6ZVHQFCqdm" (27/01/2019, 14:54).

- @ NoInvisibles: Cuenta perteneciente al documental 'Raras pero no invisibles'. 
RLCS, Revista Latina de Comunicación Social, 79, 175-205

[Investigación]10.4185/RLCS-2021-1498| ISSN 1138-5820| Año 2021

Ejemplo: "Este viernes 1 de Febrero tendrá lugar una Jornada sobre la Enfermedad de \#Huntington en Las Palmas de Gran Canaria. Os animamos a que asistais y acompañéis a parte de nuestra junta directiva que participará y asistirá a dicho evento. @FEDER_ONG @NoInvisibles RT https://t.co/Pr8CgxEPur” (29/01/2019, 12:37).

Los resultados muestran que la red de relaciones que envuelve a FEDER se asemeja a la de EURORDIS y NORD, predominando la mención como forma de interacción en los tres perfiles estudiados. En cambio, no hay ninguna referencia a la cuenta del Día Internacional de las ER, a pesar de su proximidad en el tiempo.

\subsubsection{Temas de conversación más relevantes}

En la red que rodea a FEDER también se han investigado las redes semánticas, que han permitido representar mediante una imagen cómo se interrelacionan las palabras La figura 10 ofrece una idea de las conexiones entre diferentes términos donde los nodos son los conceptos o palabras y los vínculos semánticos o de significado se muestran mediante líneas. En la imagen se puede observar que unas palabras están más conectadas que otras. Destaca la asociación (en color naranja) de FEDER con @ RarasReales (la tercera compañía biotecnológica del mundo), @NoInvisibles (el documental 'Raras pero no invisibles'), @ALBA_albinismo (asociación de ayuda a personas con albinismo), @FundacionMehuer (fundación de medicamentos huérfanos y ER), @CIBERER (Centro de Investigación Biomédica de ER), @ffpaciente (asociación de pacientes) y @ fundacionhp (fundación contra la hipertensión pulmonar).

Ejemplo: "Comienza la cuenta atrás para el Congreso \#MHER19. El acceso equitativo a los tratamientos, los avances en nuevas terapias o los casos sin diagnóstico, entre los contenidos más relevantes de esta cita, que organizamos junto a @FundacionMehuer y @FEDER_ONG. https://t.co/DXDIwKFx4Q”(01/02/2019, 9:21).

La segunda asociación de palabras presente en los tuits hace referencia a la X Carrera por la Esperanza de las personas con ER que se iba a celebrar el 3 de marzo (el color rojo), donde aparece el cantante Alejandro Sanz y su fundación de apoyo a causas solidarias, y que se completa con información sobre el cartel oficial (en azul claro).

Ejemplo: “@FEDER_ONG Lo estabais esperando y ya está aquí, el cartel oficial de la X

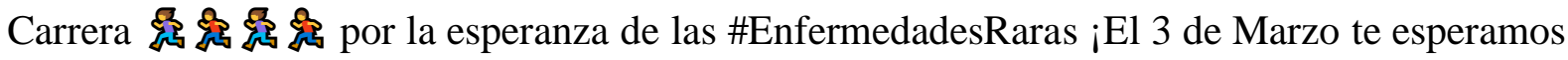
en la Casa de Campo de Madrid! \#JuntosSomosMás @AlejandroSanz $\rightarrow$ https://t.co/FgiHXCiibY https://t.co/DtHBUAGWjG” (28/01/2019, 3:04).

A su vez, otro grupo que presenta una conexión fuerte (en verde oscuro) hace referencia al Día Mundial de las ER que se celebra el 28 de febrero.

Ejemplo: "Comienza la cuenta atrás, quedan 28 días para celebrar el Día Mundial de las Enfermedades Raras https://t.co/DfY2OyCyFm” (31/01/2019, 10:34).

Otro hilo de conversación significativo (en azul oscuro) guarda relación con el programa de nómina solidaria 'Gracias a Ti', un proyecto para apoyar la labor de la Federación Española de Padres de Niños con Cáncer, la Fundación Josep Carreras y la Asociación REMAR.

Ejemplo: "Hoy con una parte de mi sueldo, aporto mi granito de arena junto al programa de nomina solidaria \#GraciasATi a seguir trabajando por un mundo mejor. Enhorabuena a las entidades @FEDER_ONG @AlzheimerEsp @FundAladina https://t.co/V378Xneouy" $(04 / 02 / 2019,11: 39)$. 
Esta iniciativa guarda relación con la Fundación ONCE y sus hoteles ILUNION ya que fueron sus trabajadores los que participaron en la actividad.

Ejemplo: "Hoy entregamos tres cheques solidariosa@FEDER_ONG, @FundAladina y a @ AlzheimerEsp por el programa de nómina solidaria \#GraciasATi al que nuestros trabajadores mes a mes aportan toda su solidaridad iiiUn aplauso para ellos!!! 808 \#GrupoSocialONCE https://t.co/uQ0YSXgpL4" (04/02/2019, 11:36).

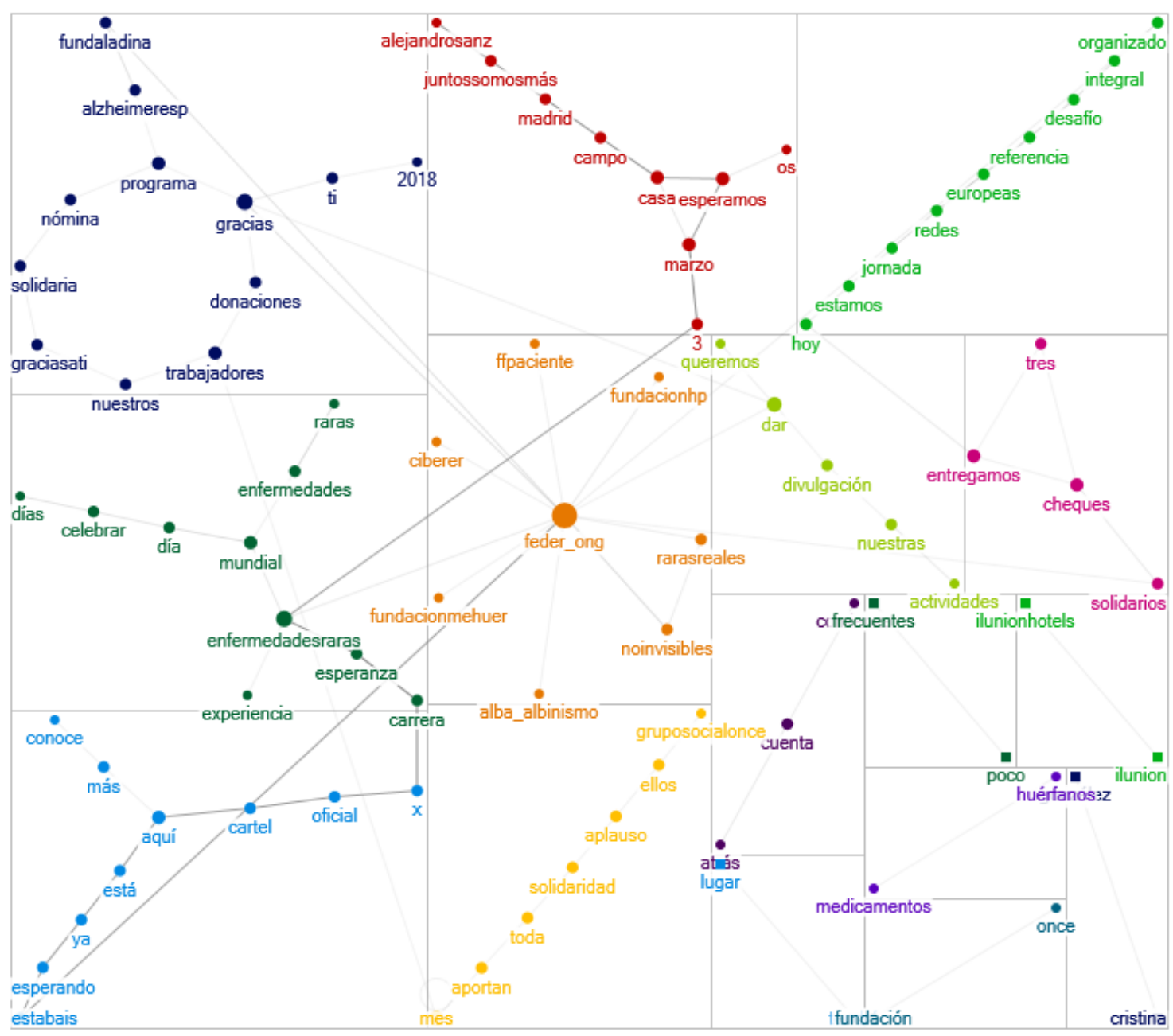

Figura 8: Hilos de conversación más relevantes en la red que rodea a FEDER

Fuente: elaboración propia

\section{Conclusiones}

En este trabajo se ha empleado el ARS como instrumento metodológico para estudiar las estructuras de red que envuelven a las asociaciones de enfermedades raras NORD, EURORDIS y FEDER e identificar a los usuarios más influyentes en Twitter. Las tres entidades disponen de perfiles que aglutinan entre 20.000 y 30.000 seguidores, una cantidad que puede resultar pequeña si se tiene en cuenta que se estima que en España existen 3 millones de individuos con ER, 30 millones de personas afectadas en Europa y otros 30 millones en EEUU. Pero disponer o no de una base amplia de seguidores sólo ofrece una orientación aproximada de la influencia que puede alcanzar un perfil y, en este sentido, resulta de mayor interés examinar métricas como el número de menciones, retuits o me gusta conseguidos por estos perfiles. En este caso, las tres cuentas tienen gran éxito entre los usuarios, ya que entre un 60 y un $80 \%$ de sus mensajes son marcados como favoritos y alcanzan tasas parecidas de 
reenvíos y de menciones. En este sentido, y en lo que se refiere al tipo de vínculos surgidos en la red, se demostró la $\mathrm{H} 2$ ya que primó el uso de la mención como modo de interacción entre los usuarios (incluyó el retuit siguiendo a Suh, et al., 2010). Este tipo de indicadores permitieron concretar la capacidad de influir de las tres cuentas analizadas ya que arrojaron información sobre el alto nivel de compromiso (engagement) y de amplificación del mensaje que consiguieron entre sus usuarios debido al interés que despiertan estas patologías y todo lo que las rodea.

El perfil más activo fue el de la federación española, a pesar de tener la décima parte de afectados por enfermedades poco frecuentes que Europa o Estados Unidos. De todas formas, las otras dos también registraron niveles adecuados de actividad, algo que es importante porque publicar contenido actualizado es la mejor forma de fidelizar usuarios y conseguir que un perfil sea influyente (Pérez, Santos y Meso, 2015).

Usando NodeXL fue posible capturar las comunidades virtuales reunidas en Twitter en torno a estos temas. Al considerar la red en términos de intermediación, cercanía o autoridad, el estudio demostró la $\mathrm{H} 1$ ya que encontró que entre los perfiles que presentaron mayor ventaja estructural en la red predominaron (por encima de los afectados) las asociaciones de pacientes, fundaciones, empresas biofarmacéuticas, centros de investigación y sus investigadores o técnicos que usaron las redes para visibilizar actividades promocionales, recaudar fondos o la difusión de la investigación.

Asimismo, quedó manifiesta la relación entre EURORDIS y NORD que, como se comentó anteriormente, unieron fuerzas en el 2010 y, entre otras cosas, acordaron promover el Día de las Enfermedades Raras como un evento de reconocimiento internacional. La proximidad en el tiempo de este hecho (quedaba menos de un mes para su celebración) lo convirtió en protagonista de gran parte de las publicaciones en ambas entidades. En FEDER, en lo que se refiere al grado de intermediación, al contrario que con EURORDIS y NORD, la cercanía del Día Internacional de las ER no alcanzó la misma relevancia ni aparecieron en los primeros puestos las otras dos organizaciones. En el caso español hay que resaltar la presencia de Sanofi España, empresa que forma parte de Genzyme Corporation, la tercera compañía biotecnológica más grande del mundo.

Si consideramos la red en términos de autoridad, las cuentas mejor conectadas en la red que envolvía a los tres perfiles estudiados fueron casi las mismas que obtuvieron un mayor grado de cercanía. Esto indica que no existieron grandes diferencias en la centralidad de la red cuando se midió el grado de prestigio.

En este estudio específico, mediante el mapeo de la red de las tres federaciones de ER, además de identificar a los usuarios de Twitter que fueron los más influyentes, la visualización mostró cómo los usuarios en diferentes grupos tuvieron conversaciones específicas en las que hablaron sobre diferentes temas. El software permitió representar mediante una imagen cómo se interrelacionaron las palabras y demostró la H3 ya que estas asociaciones emplearon las redes sociales para sensibilizar, educar e informar sobre las ER y sus problemáticas. En NORD los términos más conectados guardaron relación con la publicitación del Día Internacional de las ER y en EURORDIS fue con un simposio sobre el acceso a terapias para combatir las ER. Por último, en FEDER las palabras principales recogieron la promoción de la carrera por la esperanza de las ER donde uno de los actores más relevantes fue el cantante Alejandro Sanz y su fundación.

Para finalizar, se espera que este trabajo sirva como un recurso valioso para futuros investigadores que pueden utilizar la técnica del ARS para obtener información sobre cómo conversan los usuarios sobre ciertos temas y estudiar cómo se estructuran las redes. Hay que mencionar que este trabajo se ha 
centrado en el NodeXL pero que también hay otras herramientas que se pueden usar para el análisis de redes sociales como DMITCAT (Felt, 2016) o Gephi (Mochon, 2016), entre otras muchas.

\section{Referencias bibliográficas}

Adekunle, L. \& Adnan, M. (2016). Communicating health: media framing of Ébola outbreak in nigerian newspapers. Jurnal Komunikasi, Malaysian Journal of Communication, 32 (2), 362-380. https://ejournal.ukm.my/mjc/article/view/16486/7624

Ahmed, W. \& Lugovic, S. (2019). Social media analytics: analysis and visualisation of news diffusion using NodeXL. Online Information Review, 43(1), 149-160. https://doi.org/10.1108/OIR-03-2018$\underline{0093}$

Armayones, M., Requena, S., Gómez-Zúñiga, B., Pousada, M., \& Bañón, A. M. (2015) El uso de Facebook en asociaciones españolas de enfermedades raras: ¿cómo y para qué lo utilizan? Gaceta Sanitaria, 29(5), 335-340. https://doi.org/10.1016/i.gaceta.2015.05.007

Bakshy, E., Hofman, J. M., Mason, W. A., \& Watts, D. J. (2011) Everyone's an influencer: quantifying influence on twitter. In Proceedings of the fourth ACM international conference on Web search and data mining. 65-74. ACM.

Benetoli, A., Chen, T. F., \& Aslani, P. (2018). How patients' use of social media impacts their interactions with healthcare professionals. Patient education and counseling, 101(3), 439-444. https://doi.org/10.1016/i.pec.2017.08.015

Berkman, L. F., Kawachi, I., \& Glymour, M. M. (2014). Social epidemiology. Oxford University Press.

Brandes, U., Borgatti, S. P., \& Freeman, L. C. (2016). Maintaining the duality of closeness and betweenness centrality. Social Networks, 44, 153-159. https://doi.org/10.1016/j.socnet.2015.08.003

Camacho, I. (2009) La 'gripe A', en la prensa española. Revista Latina de Comunicación Social, 64, 827-843. http://dx.doi.org/10.4185/RLCS-64-2009-865-827-843

Castillo Esparcia, A., López Villafranca, P., Carretón Ballester, M. C. (2015) La comunicación en la red de pacientes con enfermedades raras en España. Revista Latina de Comunicación Social, 70, 673 688. http://dx.doi.org/10.4185/RLCS-2015-1065

Cavaca, A., Emerich, T., Vasconcellos, R., Santos, T. \& Oliveira, E. (2016) Diseases Neglected by the Media in Espírito Santo, Brazil in 2011-2012. PLoS Negl Trop Dis 10 (4), 1-19. http://dx.doi.org/10.1371/journal.pntd.0004662

Choi, M., Sang, Y., \& Woo Park, H. (2014) Exploring political discussions by Korean twitter users: A look at opinion leadership and homophily phenomenon. Aslib Journal of Information Management, 66(6), 582-602. https://doi.org/10.1108/AJIM-11-2012-0089

Costa, C. (2008) Medicina y salud en la prensa. Las noticias de salud en los principales diarios de Galicia. Revista Latina de Comunicación Social, 63, 15-21. http://dx.doi.org/10.4185/RLCS-632008-750-015-02 
Dalley, S. E., Buunk, A. P., \& Umit, T. (2009) Female body dissatisfaction after exposure to overweight and thin media images: The role of body mass index and neuroticism. Personality and Individual Differences, 47(1), 47-51. http://dx.doi.org/10.1016/i.paid.2009.01.044

Del Fresno García, M., Daly, A. J., \& Segado Sánchez-Cabezudo, S. (2016) Identifying the new Influences in the Internet Era: Social Media and Social Network Analysis. Revista Española de Investigaciones Sociológicas (REIS), 153(1), 23-40. http://dx.doi.org/10.5477/cis/reis.153.23

Del Fresno, M., Marqués, P., \& Paunero, D. S. (2014) Conectados por redes sociales: introducción al análisis de redes sociales y casos prácticos. Editorial UOC.

Del-Fresno-García, M. (2014). Haciendo visible lo invisible: Visualización de la estructura de las relaciones en red en Twitter por medio del Análisis de Redes Sociales. El profesional de la información, 23(3), 246-252. https://doi.org/10.3145/epi.2014.may.04

Dhar, J., Jain, A., \& Gupta, V. K. (2016) A mathematical model of news propagation on online social network and a control strategy for rumor spreading. Social Network Analysis and Mining, 6(1), 57. https://doi.org/10.1007/s13278-016-0366-5

Díaz, L. (2007) La representación de la discapacidad en los medios de comunicación. Como lograr una presencia más adecuada. Comunicación y Ciudadanía, 1. https://doi.org/10.4185/RLCS-2015-1065

Dossis, M., Amanatidis, D., \& Mylona, I. (2015) Mining Twitter Data: Case Studies with Trending Hashtags. Proceedings in ARSA-Advanced Research in Scientific Areas, (1).

Einwiller, S. A., \& Steilen, S. (2015) Handling complaints on social network sites-An analysis of complaints and complaint responses on Facebook and Twitter pages of large US companies. Public Relations Review, 41(2), 195-204. https://doi.org/10.1016/j.pubrev.2014.11.012

Emerich, T. B., Cavaca, A. G., Santos-Neto, E. T., Gentilli, V. I., \& Oliveira, A. E. (2017) Media Valuations of health journalism and health dynamics in brazilian printed media. Int'l J. Soc. Sci. Stud., 5, 31. https://doi.org/10.11114/ijsss.v5i1.1997

Felt, M. (2016) Social media and the social sciences: How researchers employ Big Data analytics. Big Data \& Society, 3(1) https://doi.org/10.1177/2053951716645828

Fink, C., Schmidt, A., Barash, V., Cameron, C., \& Macy, M. (2016) Complex contagions and the diffusion of popular Twitter hashtags in Nigeria. Social Network Analysis and Mining, 6(1), 1. https://doi.org/10.1007/s13278-015-0311-z

Freberg, K., Graham, K., McGaughey, K., \& Freberg, L. A. (2011) Who are the social media influencers? A study of public perceptions of personality. Public Relations Review, 37(1), 90-92. https://doi.org/10.1016/j.pubrev.2010.11.001

Gil de Zúñiga, H., Diehl, T., \& Ardèvol-Abreu, A. (2016) When Citizens and Journalists Interact on Twitter: Expectations of journalists' performance on social media and perceptions of media bias. Journalism Studies, 1-20. https://doi.org/10.1080/1461670X.2016.1178593

Groshek, J., \& Tandoc, E. (2017) The affordance effect: Gatekeeping and (non) reciprocal journalism on Twitter. Computers in Human Behavior, 66, 201-210. https://doi.org/10.1016/j.chb.2016.09.020 
Guo, S., Guo, X., Fang, Y., \& Vogel, D. (2017) How doctors gain social and economic returns in online health-care communities: a professional capital perspective. Journal of Management Information Systems, 34(2), 487-519. https://doi.org/10.1080/07421222.2017.1334480

Gúzman, B. \& Rodríguez, R. (2016) Comunicación y salud: la anorexia a través de la prensa. Estudios sobre el Mensaje Periodístico, 22 (2), 747-758. http://dx.doi.org/10.5209/ESMP.54233

Hansen, D., Shneiderman, B., \& Smith, M. A. (2010): Analyzing social media networks with NodeXL: Insights from a connected world. Morgan Kaufmann.

Hanusch, F. \& Bruns, A. (2017) Journalistic Branding on Twitter: A representative study of Australian journalists' profile descriptions. Digital Journalism, 5(1), 26-43. http://www.revistalatinacs.org/070/paper/1065/35es.html

Huerta, B., Fernández, B., Gallardo, C., \& Hernández, L. (2012) El rol del trabajador social en la atención de las personas afectadas por enfermedades raras. La enfermedad de Huntington. $\begin{array}{lllll}\text { Documentos } & \text { de } & \text { Trabajo } & \text { Social, } & 51,\end{array}$ https://dialnet.unirioja.es/servlet/articulo?codigo=4642268

Jacobs, R., Boyd, L., Brennan, K., Sinha, C. \& Giuliani, S. (2016) The importance of social media for patients and families affected by congenital anomalies: A Facebook cross-sectional analysis and user survey". Journal of pediatric surgery. 51(11), 1766-1771. https://doi.org/10.1016/j.jpedsurg.2016.07.008

Kuz, A., Falco, M., \& Giandini, R. (2016) Análisis de redes sociales: un caso práctico. Computación y Sistemas, 20(1), 89-106. https://dx.doi.org/10.13053/cys-20-1-2321

Kwak, H., Lee, C., Park, H., \& Moon, S. (20101) What is Twitter, a social network or a news media? In Proceedings of the 19th international conference on World wide web (pp. 591-600). ACM.

Lee, J., Agrawal, M., \& Rao, H. R. (2015) Message diffusion through social network service: The case of rumor and non-rumor related tweets during Boston bombing 2013. Information Systems Frontiers. 17(5), 997-1005. https://dx.doi.org/10.1007/s10796-015-9568-z

Leskovec, J., Adamic, L. A., \& Huberman, B. A. (2007) The dynamics of viral marketing. ACM Transactions on the Web (TWEB). 1(1), 5. https://doi.org/10.1145/1232722.1232727

Lichoti, J. K., Davies, J., Kitala, P. M., Githigia, S. M., Okoth, E., Maru, Y. \& Bishop, R. P. (2016) Social network analysis provides insights into African swine fever epidemiology. Preventive veterinary medicine, 126, 1-10. https://doi.org/10.1016/j.prevetmed.2016.01.019

Lieberman, M. (2014, March) Visualizing big data: Social network analysis. In Digital research conference (pp.

https://scholar.google.com/scholar_lookup?title=Visualizing\%20Big\%20Data\%3A\%20Social\%20 Network\%20Analysis\&author=M.\%20Lieberman\&publication_year=2014

Liu, B. (2011) Social network analysis". In Web Data Mining (pp. 269-309). Springer Berlin Heidelberg. 
López-Villafranca, P. (2016) Estudio de la presencia de los gabinetes de comunicación en las organizaciones de pacientes con enfermedades raras en España. Revista internacional de Relaciones Públicas. 11(6), 27-46. http://dx.doi.org/10.5783/RIRP-11-2016-03-27-46.

López-Villafranca, P. \& Castillo-Esparcia, A. (2018) El encuadre de las enfermedades raras en los medios de comunicación españoles. Observatorio $\left(O B S^{*}\right), \quad 12(2), \quad 136-$ 155. https://doi.org/10.15847/obsobs12220181074

Meijer, A. J. \& Torenvlied, R. (2016) Social media and the new organization of government communications: An empirical analysis of Twitter usage by the Dutch police. The American Review of Public Administration. 46(2), 143-161. https://doi.org/10.1177/0275074014551381

Mochón, M. C. (2016) Social network analysis and big data tools applied to the systemic risk supervision. Ijimai. 3(6), 34-37. https://doi.org/10.9781/ijimai.2016.365

Observatorio Nacional de las Telecomunicaciones y de la Sociedad de la Información (ONTSI) (2016): Los ciudadanos ante la e-Sanidad. Estudio sobre opiniones y expectativas de los ciudadanos sobre el uso y aplicación de las TIC en el ámbito sanitario. https://www.ontsi.red.es/ontsi/sites/ontsi/files/los ciudadanos ante la e-sanidad.pdf

O'Keeffe, G. S. \& Clarke-Pearson, K. (2011) The impact of social media on children, adolescents, and families. Pediatrics, 127(4), 800-804. https://doi.org/10.1542/peds.2011-0054

Pérez Dasilva, J., Santos Díez, M. T. \& Meso Ayerdi, K. (2015) Radio y redes sociales: el caso de los programas deportivos en Twitter. Revista Latina de Comunicación Social, (70), 141-155. https://doi.org/10.4185/RLCS-2015-1039

Pérez Dasilva, J., Meso Ayerdi, K. \& Mendiguren Galdospín, T. (2018) ¿Dialogan los líderes políticos españoles en Twitter con los medios de comunicación y periodistas? Comunicación y Sociedad, 31(3), 299-316. https://doi.org/10.15581/003.31.3.299-315

Peñafiel, C. Camacho, I. Aiestaran, A. Ronco, M. \& Echegaray L. (2014) La divulgación de la información de salud: un reto de confianza entre los diferentes sectores implicados. Revista Latina de Comunicación Social, 69, 135- 151. https://doi.org/10.4185/RLCS-2014-1005

Pfeffer, J., Zorbach, T. \& Carley, K. M. (2014) Understanding online firestorms: Negative word-ofmouth dynamics in social media networks. Journal of Marketing Communications, 20(1-2), $117-$ 128. https://doi.org/10.15581/003.31.3.299-315

Posada, M., Martín-Arribas, C., Ramírez, A., Villaverde, A. y Abaitua, I. (2008) Enfermedades raras. Concepto, epidemiología y situación actual en España. Anales del Sistema Sanitario de Navarra, 31(2). http://scielo.isciii.es/pdf/asisna/v31s2/original2.pdf

Roberts, A. \& Good, E. (2010) Media images and female body dissatisfaction: The moderating effects of the Five-Factor traits". Eating Behaviours, 4(11), 211-216. USA, Indiana University. http://dx.doi.org/10.1016/j.eatbeh.2010.04.002

Salinas, S. O., Trujillo, J. M. P. \& Montero, R. A. S. (2017) Election analysis in Colombia and Venezuela 2015 through sentiment analysis and Twitter. Sistemas \& Telemática, 14(39), 57-70. https://doi.org/10.18046/syt.v14i39.2349 
Sánchez Hernández, F. (2016) Análisis sobre los contenidos de enfermedades raras en la prensa escrita española. Revista Española de Comunicación en Salud, 242-260. http://dx.doi.org/10.20318/recs.2016.3450

Santos, M. T. y Pérez Dasilva, J. A. (2018) Las enfermedades raras en la red: El caso de FEDER en Twitter. En: IX Congreso Internacional de Ciberperiodismo. UPV-EHU, Bilbao.

Santos, M. T. y Pérez Dasilva, J. A. (2019) Las enfermedades raras y su representación en la prensa española". Palabra Clave 22(1), 254-287. https://doi.org/10.5294/pacla.2019.22.1.10

Schroeder, R. (2016) Online Entertainment the Globalization of On-Screen Sociability: Social Media and Tethered Togetherness. International Journal of Communication, 10, 18. https://doi.org/10.1932-8036/20160005

Schumacher, K., Stringer, K., Donohue, J., Yu, S., Shaver, A., Caruthers, R., Zikmund-Fisher, B., Fifer Goldberg, C. \& Russell, M. (2014) Social media methods for studying rare diseases. Pediatrics, 133(5), 1345-1353. https://doi.org/10.1542/peds.2013-2966.

Scott, J. (2012): Social network analysis. Sage.

Shin, J., Jian, L., Driscoll, K., \& Bar, F. (2016) Political rumoring on Twitter during the 2012 US presidential election: Rumor diffusion and correction. New media \& society, 1, 22.

Shu, W., \& Chuang, Y. H. (2011) The perceived benefits of six-degree-separation social networks. Internet Research, 21(1), 26-45. https://doi.org/10.1108/10662241111104866

Smith, M. A. (2014) NodeXL: Simple network analysis for social media. In Encyclopedia of Social Network Analysis and Mining (pp. 1153-1170). Springer New York.

Solves. J. \& Bañón A. (2014) Las Enfermedades Raras en los medios. Informe Enero-Marzo Observatoria sobre Enfermedades Raras (OBSER).

Suh, B., Hong, L., Pirolli, P., \& Chi, E. H. (2010) Want to be retweeted? large scale analytics on factors impacting retweet in twitter network. In 2010 IEEE Second International Conference on Social Computing (pp. 177-184). IEEE.

Terrón, J. L. (2012) El tratamiento del VIH-sida en los periódicos españoles, una investigación colaborativa. Revista de Comunicación y $\quad$ salud 1 (1), 4-17. https://dialnet.unirioja.es/ejemplar/279774

Verweij, P. (2012) Twitter links between politicians and journalists. Journalism Practice, 6(5-6), 680691. https://doi.org/10.1080/17512786.2012.667272

Villa, Á., Llombart, E., Solanillo, F., Queralt, R., \& Castillo, S. (2014) Las enfermedades raras en los medios. Observatorio sobre enfermedades raras (OBSER). https://www.enfermedadesraras.org/images/feder/Documentos ultimas noticias/INFORME OBSER 32014 MEDIOS TE RCER_TRIMESTRE.pdf 
Williams, S. A., Terras, M. M., \& Warwick, C. (2013) What do people study when they study Twitter? Classifying Twitter related academic papers. Journal of Documentation, 69(3), 384-410. https://doi.org/10.1108/JD-03-2012-0027

Wu, S., Hofman, J. M., Mason, W. A., \& Watts, D. J. (2011) Who says what to whom on twitter. In Proceedings of the 20th international conference on World wide web (pp. 705-714). ACM.

Yoo, J. \& Kim, J. (2012) Obesity in the new media. A content analysis of obesity videos on youtube. Health Communication 27, 86-97. https://doi.org/10.1080/10410236.2011.569003

Zambrano, G. R., Tomalá, J. R., \& Albiño, W. A. (2016) Feeling's analysis using the Twitter API. International Journal of Innovation and Applied Studies, 16(4), 814. http://www.ijias.issrjournals.org/abstract.php?article=IJIAS-16-043-08

Zappavigna, M. (2015) Searchable talk: the linguistic functions of hashtags. Social Semiotics, 25(3), 274-291. https://doi.org/10.1080/10350330.2014.996948

\section{AUTORES:}

\section{Jesús Ángel Pérez Dasilva}

En la actualidad es profesor agregado en el departamento de Periodismo II de la Facultad de Ciencias Sociales y de la Comunicación de la Universidad del País Vasco donde imparte la asignatura Redacción Ciberperiodística. En lo referido a gestión, ha sido vicedecano de Movilidad Internacional de la Facultad y director del Máster Universitario de Comunicación Social. Como investigador, ha realizado dos estancias investigadoras en la Universidad de Cambridge (2012) y la Universidad de Sevilla (2010). También ha realizado movilidades docentes dentro del marco Erasmus a las Universidades de Wroclaw (2017), do Minho (2016), Oporto (2015), Beira Interior (2013), Trieste (2011) y Nova de Lisboa (2010). Además, ha participado en una docena de proyectos de investigación y es autor de una veintena de artículos científicos publicados en revistas de prestigio incluidas en bases de datos como JCR, SCOPUS o Dice-Cindoc. Actualmente, es miembro del grupo consolidado de investigación Gureiker y del proyecto "Audiencias activas y viralización y transformación de los mensajes periodísticos" (CSO2015-64955-C4-4-R), financiado por el Plan Nacional del I+D+i, del Ministerio de Economía y Competitividad, y por el Fondo Europeo de Desarrollo Regional (FEDER).

jesusangel.perez@ehu.eus

Índice H: 9

ORCID: http://orcid.org/0000-0002-3383-4859

Google Schoolar: http://scholar.google.es/citations?user=NANienYAAAAJ\&hl=es\&oi=ao

\section{$M^{a}$ Teresa Santos}

En la actualidad es profesora Titular de Universidad en la Facultad de Ciencias Sociales y Comunicación UPV/EHU. Las líneas de investigación se centran en medios locales (prensa, radio, televisión), comunicación, salud y periodismo digital. Es autora de varios libros y artículos como: La radio latina en España. Un medio para la integración (Perfiles Latinoamericanos 2016), Treatment of cannabis in the Spanish press (Cuadernos Info 2017) y Redes sociales y evangelización: el caso de las diócesis españolas en "Facebook" (Estudios Mensaje Periodístico 2017), Features and Dimensions of Health Care Journalism: A Case Study on Spanish Free Magazines (Sage Open, 2017), Therapeutic cannabis in the Spanish newspapers (Estudios Mensaje Periodístico, 2018) y Rare Diseases and their Representation in the Spanish Press (Palabra Clave, 2019) entre otros.

mariateresa.santos@ehu.eus 


\section{Índice H: 7}

ORCID: https://orcid.org/0000-0002-2494-3279

Google Schoolar: https://scholar.google.es/citations?user=h2zcuPsAAAAJ\&hl=es

\section{Koldobika Meso Ayerdi}

En la actualidad es profesor titular de la Facultad de Ciencias Sociales y de la Comunicación de la Universidad del País Vasco, donde imparte las asignaturas Redacción ciberperiodística y Bases teóricas y metodología de la investigación en ciberperiodismo en el Master de Investigación Social de la UPV. También ha impartido materias como Modelos de los Medios de Comunicación e Introducción al Periodismo. Es autor de varios libros sobre periodismo en Internet y ha publicado una veintena de artículos en revistas como Estudios del Mensaje Periodístico, Zer, Análisi, y Latina. Actualmente es el director del Departamento de Periodismo II de la UPV-EHU y dirige el proyecto financiado por el Ministerio de Economía y Competitividad titulado "Audiencias activas y periodismo: análisis de la calidad y la regulación de los contenidos elaborados por los usuarios", con referencia CSO2012-39518C04-03.

koldo.meso@ehu.eus

Índice H: 16

ORCID: http://orcid.org/0000-0002-0400-133X

Google Schoolar: http://scholar.google.es/citations?user=daFlEQIAAAAJ\&hl=es\&oi=ao 


\section{Texto para el video (formulario 500)}

En este trabajo se ha empleado el ARS como instrumento metodológico para estudiar las estructuras de red que envuelven a las asociaciones de enfermedades raras NORD, EURORDIS y FEDER e identificar a los usuarios más influyentes en Twitter.

Las tres entidades disponen de perfiles que aglutinan entre 20.000 y 30.000 seguidores, una cantidad que puede resultar pequeña si se tiene en cuenta que se estima que en España existen 3 millones de individuos con ER, 30 millones de personas afectadas en Europa y otros 30 millones en EEUU. Pero disponer o no de una base amplia de seguidores sólo ofrece una orientación aproximada de la influencia que puede alcanzar un perfil y, en este sentido, resulta de mayor interés examinar métricas como el número de menciones, retuits o me gusta conseguidos por estos perfiles. En este caso, las tres cuentas tienen gran éxito entre los usuarios, ya que entre un 60 y un $80 \%$ de sus mensajes son marcados como favoritos y alcanzan tasas parecidas de reenvíos y de menciones. En este sentido, y en lo que se refiere al tipo de vínculos surgidos en la red, primó el uso de la mención como modo de interacción entre los usuarios (incluyó el retuit siguiendo a Suh, et al., 2010). Este tipo de indicadores permitieron concretar la capacidad de influir de las tres cuentas analizadas ya que arrojaron información sobre el alto nivel de compromiso (engagement) y de amplificación del mensaje que consiguieron entre sus usuarios debido al interés que despiertan estas patologías y todo lo que las rodea.

El perfil más activo fue el de la federación española, a pesar de tener la décima parte de afectados por enfermedades poco frecuentes que Europa o Estados Unidos. De todas formas, las otras dos también registraron niveles adecuados de actividad, algo que es importante porque publicar contenido actualizado es la mejor forma de fidelizar usuarios y conseguir que un perfil sea influyente (Pérez, Santos y Meso, 2015).

Usando NodeXL fue posible capturar las comunidades virtuales reunidas en Twitter en torno a estos temas. Al considerar la red en términos de intermediación, cercanía o autoridad, el estudio encontró que entre los perfiles que presentaron mayor ventaja estructural en la red predominaron (por encima de los afectados) las asociaciones de pacientes, fundaciones, empresas biofarmacéuticas, centros de investigación y sus investigadores o técnicos que usaron las redes para visibilizar actividades promocionales, recaudar fondos o la difusión de la investigación.

Asimismo, quedó manifiesta la relación entre EURORDIS y NORD que, como se comentó anteriormente, unieron fuerzas en el $2010 \mathrm{y}$, entre otras cosas, acordaron promover el Día de las Enfermedades Raras como un evento de reconocimiento internacional. La proximidad en el tiempo de este hecho (quedaba menos de un mes para su celebración) lo convirtió en protagonista de gran parte de las publicaciones en ambas entidades. En FEDER, en lo que se refiere al grado de intermediación, al contrario que con EURORDIS y NORD, la cercanía del Día Internacional de las ER no alcanzó la misma relevancia ni aparecieron en los primeros puestos las otras dos organizaciones. En el caso español hay que resaltar la presencia de Sanofi España, empresa que forma parte de Genzyme Corporation, la tercera compañía biotecnológica más grande del mundo.

Si consideramos la red en términos de autoridad, las cuentas mejor conectadas en la red que envolvía a los tres perfiles estudiados fueron casi las mismas que obtuvieron un mayor grado de cercanía. Esto indica que no existieron grandes diferencias en la centralidad de la red cuando se midió el grado de prestigio.

En este estudio específico, mediante el mapeo de la red de las tres federaciones de ER, además de identificar a los usuarios de Twitter que fueron los más influyentes, la visualización mostró cómo los 
usuarios en diferentes grupos tuvieron conversaciones específicas en las que hablaron sobre diferentes temas. El software permitió representar mediante una imagen cómo se interrelacionaron las palabras y demostró que estas asociaciones emplearon las redes sociales para sensibilizar, educar e informar sobre las ER y sus problemáticas. En NORD los términos más conectados guardaron relación con la publicitación del Día Internacional de las ER y en EURORDIS fue con un simposio sobre el acceso a terapias para combatir las ER. Por último, en FEDER las palabras principales recogieron la promoción de la carrera por la esperanza de las ER donde uno de los actores más relevantes fue el cantante Alejandro Sanz y su fundación.

Para finalizar, se espera que este trabajo sirva como un recurso valioso para futuros investigadores que pueden utilizar la técnica del ARS para obtener información sobre cómo conversan los usuarios sobre ciertos temas y estudiar cómo se estructuran las redes. Hay que mencionar que este trabajo se ha centrado en el NodeXL pero que también hay otras herramientas que se pueden usar para el análisis de redes sociales como DMITCAT (Felt, 2016) o Gephi (Mochon, 2016), entre otras muchas. 